\title{
EVERYONE LEFT
}

By

Graeme Ross Kennedy

Bachelor of Architectural Studies in Design, Carleton University, Spring 2014

A Major Research Paper

Presented to Ryerson University

in partial fulfillment of the requirements for the degree of

\author{
Master of Planning \\ in \\ Urban Development
}

Toronto, Ontario, Canada, 2019

(C) Graeme Ross Kennedy 2019 


\section{Author's Declaration for Electronic Submission of a MRP}

I hereby declare that I am the sole author of this MRP. This is a true copy of the MRP, including any required final revisions.

I authorize Ryerson University to lend this paper to other institutions or individuals for the purpose of scholarly research.

I further authorize Ryerson University to reproduce this MRP by photocopying or by other means, in total or in part, at the request of other institutions or individuals for the purpose of scholarly research.

I understand that my MRP may be made electronically available to the public. 


\title{
EVERYONE LEFT
}

(C) Graeme Ross Kennedy, 2019

Master of Planning

In

Urban Development

Ryerson University

\begin{abstract}
The importance of inscribing maintenance and continuity — disruption mitigation — measures into Smart City technology has long been an overlooked topic in proposals and procurement processes, hampered in part by lack of legibility, advocacy, and community capacity for addressing innovation. The paper analyzed four select cases from the Top 20 finalists of Infrastructure Canada's Smart Cities Challenge by asking the question, 'what happens when innovators move on?'. Cases focused on the themes of intergenerational knowledge, food security, test-bed urbanism, and disaster response. Case specific examinations were synthesized into broader explorations regarding addressing underlying infrastructure, labour force availability \& training, and the role of government and planners in these projects. Finally, future research recommendations discuss how to package site specific maintenance and continuity measures into Smart City projects such that communities are equipped to assume systems from the innovators.
\end{abstract}

Key words: Smart City development; urban planning innovation; infrastructure systems disruption. 


\section{Acknowledgements}

Thank you to my supervisor, Dr. Pamela Robinson. This research all arose due to your support of weird ideas and creating a curriculum that allowed me to splash around in the dark twisted world that we call sustainability. Thank you for allowing me to chase sheep and dive down rabbit holes.

Thank you to my second reader, Charles Finlay, for taking the time to review my MRP. I deeply appreciate your time and feedback, as well as the public leadership role that you have taken regarding waterfront Smart City discourse in Toronto.

To my colleagues and professors at Ryerson University's School of Urban and Regional Planning, you have inspired and motivated me. Thank you for an incredibly rewarding two years of learning, growing, and planning.

Finally, This MRP was funded, in part, through research grants held by Dr. Pamela Robinson (SSHRC Grants: 892-2018-0046 and 895-2012-1023). The Social Sciences and Humanities Research Council has played, and will continue to play, a vital role in supporting research in this field and many other fields. SHHRC-funded research builds understanding that can help change the world. The council's ongoing support of students specifically at Ryerson's School of Urban and Regional Planning cannot be recognized enough. 


\section{Dedication}

\begin{tabular}{|lll|}
\hline & & \\
To those who & and to those who & and to those on \\
$\begin{array}{lll}\text { lit the way; } & \text { picked up the torch; } & \text { the frontline; } \\
+ \text { Lovelace } & + \text { Bjirke } & + \text { Robinson } \\
+ \text { Meadows } & + \text { Lepore } & + \text { Lister } \\
+ \text { Ostrem } & + \text { Mattern } & + \text { Wylie }\end{array}$
\end{tabular}

And to all those working on behalf of everyone left, for the past 172 years, and the next 172 years.

\begin{tabular}{lr}
\hline \\
$\begin{array}{l}\text { I understand about indecision } \\
\text { But I don't care if I get behind }\end{array}$
\end{tabular}




\section{Table of Contents}

Author's Declaration for Electronic Submission of a MRP ii

ABSTRACT

Acknowledgements $\quad$ iv

Dedication: $\quad$ V

Table of Contents

List of Appendices $\quad$ vi

Ch 1 Introduction 1

$\begin{array}{ll}\text { Ch } 2 \text { Literature review } & 7\end{array}$

$\begin{array}{ll}\text { Ch } 3 \text { Method } & 20\end{array}$

Ch 4 Case Analysis $\quad 23$

Ch 5 Conclusions $\quad 40$

$\begin{array}{lr}\text { Postscript } & 51\end{array}$

Appendices $\quad 52$

$\begin{array}{lr}\text { References } & 60\end{array}$

List of Appendices

Appendix 1 - Top Twenty Smart Cities Challenge Finalists 52

Appendix 2 - Places to Intervene in a System 53

Appendix 3 - Types of Systems \& Scales of Systems

Appendix 4 - Rust, Dust, Cracks, \& Corruption 55

Appendix 5 - SCC Entries Selection Flow Chart 56

Appendix 6 - SCC Entries $\quad 57$

Appendix 7 - City of Richmond - Proposed Emergency Response System 58

Appendix 8 - Kurzweil’s Model 59 


\section{Ch 1 Introduction}

The Smart City is different from other cities - every city is different from its contemporaries - and its social-technical systems are different too. To review: a city is an organized form of human settlement, and Smart, in this context, refers to the use of interconnected and interdependent communications technologies in the pursuit of, as of yet, unrealized efficiencies. Some would deem these efficiencies impossible for less than Smart technologies within a specific locale. Therefore, a Smart City is a human settlement in the process of adopting new Smart infrastructure, uniting its members under the promise of progress.

When Smart Cities rise out of their existing state, they do so by telling the stories of their future; stories designed to suggest that all participants will flourish, stories of the end product, stories of a better life for all involved (Lepore, 2019; Dinerstein, 2006). Between then and now is a process of conversion, renewal, and change. This process can be peaceful or volatile. It involves unwinding old systems and deploying new ones. To be sustainable, the Smart City requires maintainable technologies that can change cities and a community that wants to change.

Technology is transformative. New innovations carry enormous potential to fundamentally alter our cities. The results of previous waves still carry us forward today. Novel technologies at many points in history have changed how we build cities and how we measure them. The collective successes or failures of new technologies informed future revelations regarding how we design cities. The mass availability of automobiles and the construction of limited access interstate highways not only changed the radius of cities but material spillover impacts on the cities that bordered interchanges. Kunstler (1993) documented how local architectural vernaculars of the Main Street lost prominence as large corporations built cookiecutter complexes close to highways on the urban fringe of a city, a trend Lewis' (1995) dubbed the 'Galactic City'. Similarly, successive advancements in elevator technology and engineering materially altered achievable densities in cities with new buildings capable of reaching ever higher heights (Harford, 2017). Our ability to map and measure data to uncover patterns also lead to breakthroughs in citywide planning trends, as per John Snow's mapping of cholera in 1854 (Brasuell, 2017) and William Whyte's (1980) use of video and time lapse photography. Whyte's data was used to inform the design of future public spaces. Commenting in the Ontario Planning Journal, Noble extends the results of planning research into future planning thought, stating,

"who knew how much people liked to move their chairs around? [...] These new abilities create new responsibilities. One is the responsibility of all levels of government to work with community partners to examine the costs and benefits of the technologies and to develop the next generation of digital governance structures. Another is the responsibility of planners to use the information wisely to create a better city" (2018, p. 4)

How cities evolve through adopting new technologies has been at the periphery of the planning profession for quite some time. Where the Smart City is different from previous waves of change is in the interconnected nature of the systems being proposed. New technologies are both physical objects and digital measurement systems capable of responding to newly discovered patterns. This changes renewal in a way that has not impacted urban domains in such an explosive manner since the Industrial Revolution. Many of the new technologies being deployed in cities modify service delivery, underlying 
infrastructure(s), and the collective operations of the urban domain. Uber (Express Pool), Lyft, and even Bird scooters purport to be a more efficient alternative to the delivery of transit services through subsidizing fares with venture capital (Kosoff, 2018). Underlying infrastructure, normally procured by government, has recently seen the emergence of installations whose embedded interconnectivity enables the advancement of surveillance-based capitalism, with municipalities procuring platforms like Shotspotter (Patton, 2018), replacing phone boxes with Smart kiosks (Atkins, 2018), and General Electric's camera-equipped streetlights in San Diego (Pandey, 2019). Finally, there are examples of large firms acting to replace the functions of government itself with Bitcoin and Ethereum printing their own currency (Hartshorn, 2018), Amazon (a supply chain technology company) running a (reverse) municipal RFP process for their HQ2 (Thompson, 2018b), and Sidewalk Labs operating as if, "assuming the patina of lawmaking authority typically exercised by local government" (Pandey, 2019). What connects all of these disparate products is their use of interconnected communications technologies ("ICTs") as a connective fabric that can be layered on top of cities in pursuit of higher efficiency, higher leverage, or higher value forms of urban practice.

The emergence of Smart Cities as an urban planning trend has been met with curiosity, enthusiasm, uncertainty, and concern. Voigt stated that this trend, "compromises our ability to effectively prepare the communities we serve" (2018, p. 22) further stating that the Smart City, "represents city planning in a social, cultural, and urban design framework with no historic precedent or contextual touchstone." (Ibid.). If handled correctly the transformative promise of the technologies that comprise the Smart City trend carry the potential to empower a paradigm shift in city building.

Due to the multifaceted and complex nature of the systems being deployed, universal consensus on the exact definition of a Smart City is unlikely to be reached. Cruz and Sarmento (2017) outline three definitions for defining Smart City innovations as a spectrum that measures the level of risk and uncertainty involved in Smart City projects. Clarke (2018) and Kaner (2019) speak to the level of service delivery, state capacity, and community engagement that define successive waves of digital government and Smart City initiatives. 'Smart City technology' within the Smart City concept is, itself, an incredibly broad and nebulous category. For the purposes of this research, Smart City technology will be narrowed to focus on technologies that combine interconnectivity with existing physical infrastructure or novel iterations thereof, for it is found that it is difficult to engage in meaningful city building with just an app. Emerging Smart City technologies have far reaching implications for the quality of our day-to-day civic operations, our governance structures, and our cultural continuity. Collectively these authors discuss how risk, uncertainty, and funding issues are mitigated through procurement agreements between the public and private sectors. Discourse surrounding this trend has expressed apprehension over governance and implementation strategies, especially when governments turn to the private sector to collaborate on these changes.

Funding new Smart Cities projects through Public Private Partnerships ("P3s") is not outside of the norm. Private companies and citizens play a significant role in developing and deploying new 'smart' infrastructures in many different cities. Yet, Smart City technology companies do not just appear, and existing infrastructure companies do not invest heavily in Smart City research and development. Many of the products being proposed for Smart City applications are the result of upmarket disruption (Christensen, 2016) from the consumer products and mobile applications sectors where these ideas are 
developed. Private sector technology actors have shown a propensity for not maintaining their products on a long term basis, either through lack of support or planned obsolescence. For example Google's acquisition of Revolv remotely disabled all of their existing smart thermostats (Statt, 2016) with owners commenting,

"On May 15th, my house will stop working. My landscape lighting will stop turning on and off, my security lights will stop reacting to motion, and my home made vacation burglar deterrent will stop working. This is a conscious intentional decision by Google/Nest [...] Is the era of IoT [Internet of Things] bringing an end to the concept of ownership? Are we just buying intentionally temporary hardware?" (Gilbert, 2016)

As more public funding is dedicated towards Smart City projects, and these technology vendors pivot into operating in the Smart Cities context, it becomes the fiduciary obligation of urban planners, on top of the protection of public interest, to ensure that the technological solutions being proposed are maintainable, sustainable, and resilient towards long term disruption via deprecation by the original vendor.

Deprecation adds additional factors to how urban operations can be disrupted, joining expected wear and tear, and infrastructure/systems volatility (disruption due to man-made or natural acute shocks or chronic stresses). Mitigating the fallout of technological disruption in cities is crucial to ensuring the longevity and social cohesion of the urban realm. This is especially pertinent when disruptive city-building actors opt for tabula rasa approaches to civic infrastructure renewal. For example the Sidewalk Labs project stating that they were building, "the world's first neighbourhood built from the internet up" (Doctoroff, 2017), which positions the project in a way that allows them to discard the embodied wisdom found within stable and sustainable cities that were not built from the internet up. The prevailing thinking for innovators acting in this domain is to suggest that 'Smart Cities' and new infrastructure systems require non-traditional approaches and thus are outside of the thinking of incumbent city-building initiatives, both in the public and private sectors. The shortsighted nature of this thinking ignores the intergenerational wisdom inscribed into our civic infrastructure through open specifications and the training of our 'maintainer' class labour (Bliss, 2016), the complex and interwoven systems within which renewal takes place, and the innovator's own susceptibility to disruption, erasure, or product deprecation. Smart City systems built on closed software architectures, proprietary hardware, or that are fulfilled by offsite 'cloud' services carry with them a lack of oversight and community involvement, and thus agency or knowledge contribution from community-based actors that will be forced to repair systems if the vendor fails to support their infrastructure products, if such a repair is even possible. The willful subversion of DIYmodifications, professional training programs, and industry best practice means that if problems occur, the ability to repair, maintain, and continue use of the technology lies solely with the innovator themselves.

The spectre of disruption and deprecation has not one but two factors to which responses are required prior to systems change. The first being how maintenance and continuity, or mechanisms thereof, are being inscribed as requirements into the new civic systems being proposed. The second factor examines how maintenance and continuity of the community is being included, reinforced, empowered, or maintained through use of, or despite, new technologies. The combination of these two factors collectively illustrates a community's capacity for the new technology and its deployment within the their municipality. 
Maintainability, sustainability, and resilience should not solely be required of the technology being proposed for systems change but also the communities within which new systems will be deployed. New technologies modify behaviours and trigger cultural shifts that change how people interact with each other, their communities, and government. Cultural shifts can be both positive and negative. In a manner similar to how our infrastructure needs to be maintained, our relationship to our communities and the cultural narrative of our cultures deserves similar attention. As the creators of new technologies push for increased efficiencies in our urban domains it is incumbent upon urban planners to weigh the cultural impacts of new technologies. Under consideration are how they can further cultural continuity, maintain traditional knowledge \& customs, or permanently alter behaviours beyond repair. How our collective wisdom, stories, culture, language, and governance structures are continued or changed by new technologies must be considered to the same extent as the technologies themselves.

The notion of maintainability or 'maintenance' can seem quite banal but, if overlooked, can have a severe impact on the quality of life of a community's current and future members. When communities adopt new technologies, either through force or monitored systems change, disruption occurs. Old products, methods of practice, and infrastructure will be replaced with new systems. To ensure that failure of a new system will not materially damage a community it is critical that Smart City projects be evaluated for how long term maintenance, sustainability, and resilience practices have been inscribed into the product to ensure long-term continuity. If code is not auditable, editable, or accessible or if hardware is locked, proprietary, or inaccessible to repair then, it is argued, a system is incapable of outliving its creator. If unique wisdom is not shared, if cultural practices cannot be passed on, if languages are not learned and used, a community becomes incapable of surviving its citizens. In such instances the technologies being proposed may not be in the best interest of the public or appropriate for receiving public funds.

How cities adapt and evolve as well as how development is guided is reliant upon visioning exercises that illustrate a path to the future that residents want to see. The long term development of our cities can guided decision-making regarding which technologies will be helpful in achieving this vision and which can be discarded. In this way, the planning of future urban development and the procurement of new technologies are linked in a recursive feedback loop, with awareness of new technologies unlocking new possibilities for cities and the visioning of a city's future highlighting problems worth solving with new technologies.

Innovations in educational infrastructure, transit \& mobility, food production, logistics, and probabilistic modelling (colloquially 'artificial intelligence') can all illustrate new possible futures for our cities. In many ways it falls upon planners to consider how these technologies mesh with our existing civic narrative when considering their adaption and how best to adapt our existing services to accomodate or mitigate the impacts of their (un)expected arrival.

In November 2017 the Government of Canada challenged municipalities across the country to engage their communities to prepare entries for Infrastructure Canada's Smart Cities Challenge. Entries needed to propose solutions for addressing a problem being faced by their community through the use of innovative technologies and display what role community engagement had in guiding their proposal. The Top 20 finalists were announced on June 1st, 2018. Each of the twenty communities received $\$ 250,000$ to put 
towards developing their final proposals due March 5th, 2019, with the winners due to be announced in Spring 2019.

The Smart Cities Challenge is an opportunity for communities and municipalities across the country to procure cutting edge technology that may otherwise be cost-prohibitive. Posing such an opportunity as a 'challenge' signals two things. The first being that these projects are expensive to implement and the Federal government is not willing or able to fund the deployment of new technologies in every interested community. Second, that interested communities must display public sector knowledge of new technologies and the capacity to create a successful proposal. Proposals sent in by communities of all sizes encompass a range of interconnected problems to be solved at complex scales of operation. The submissions collectively encompass a broad spectrum of Smart City innovative technologies, systems, and governance structures.

This research was conducted through Fall 2018 and Winter 2019 prior to the announcement of the Smart Cities Challenge winners. At this time, analysis of cases selected from the Top 20 is timely, relevant, and, currently, preempts the awarding of funds. Many of the proposals are advocating for transformative technologies that have not yet operated at a mass scale, or at the same level of community reliance, where best practice can be established. It is hoped that this research will contribute towards discourse on which projects are best situated to received funding and help to inform future award processes and evaluation criteria. The examination of how questions were asked and how respondents answered is coupled with commentary on what was not included in responses. The consideration of long-term sustainability requirements of emerging technologies poses the potential to ensure that government funding is put towards lasting positive systems change.

Analysis of Smart Cities Challenge entries will explore potential points of failure where continuity and maintainability are being overlooked. This paper will seek to identify some of these problems and make recommendations to increase the likelihood of continuity. To be successful, the adoption of new technologies needs to be aligned with civic goals. Investment of time, space, and resources in the procured systems needs to be justified by the problem it is addressing. The impact of disrupting existing services in order to deploy new one needs to be weighed against the benefit of the new system.

To be sure, these are new concepts that bring with them new ways of designing, building, and operating cities. Farber (2010) argues that the conventional tools of professional planning do not equip planners to cope with the potentially disastrous results of disruption. This may not hold to be true. Cities are still cities. New technologies and novel forms of renewal have always been a part of the planning vocabulary, just under terms of reference. This research benefits planners by outlining potential weaknesses in Smart Cities systems and advancing ideas for how best to address them with conventional planning tools of engagement, governance, policy, and process. The focus of this research will help planners to situate themselves within a Smart City context and operate using a framework that highlights urban continuity and sustainability issues that might be overlooked. In instances such as the aforementioned, the Canadian Institute of Planners Statement of Values asserts what planners should value when approaching Smart City projects but does not define how best to ensure that those values are protected. Including the first and fourth points; to respect and integrate the needs of future generations, and to recognize and react positively to uncertainty must be prominent in the planner's mind when analyzing new technology driven 
solutions and their integration into our cities. This line of research provides one vector by which ensure system and cultural continuity through the acute shocks and chronic stresses of systems change to Smart City modes of practice.

The proposed research will examine maintenance and sustainability in Smart City projects by using Infrastructure Canada's Smart City Competition applicants as the body of case studies to answer the question; what happens when innovators move on? The question itself is impossible to comprehensively answer. Rather it excites analysis of Smart City projects in a manner that splits inquiry into two parts; what are we currently doing to support continuity, maintainability, and sustainability in Smart City projects and what could we be doing?

The intent is to bridge the gap between examination of domain-specific disruption and its implications on Smart City projects as a means by which to design a framework for ensuring that a proposed site is capable of resilience toward the adverse impacts of disruption during and long-past its integration. By synthesizing these two realms of practice and research one can begin to develop a toolkit that examines and provides guidance with regard to the interweaving roles that policymakers, disruptive actors, and maintainers play in advancing the utility of our cities in combination with maintaining social cohesion. The end goal of this study is to develop suggestions for how to equip planners to appropriately respond to civic disruption. Planners cannot simply weather the storm, we need to learn how to dance in the rain. 


\section{Ch 2 Literature review}

The question, 'what happens when innovators move on?' was parsed into more targeted and specific lenses of analysis which each draw upon research from multiple disciplines. The topics of focus which emerged are the themes of Innovation Policy \& Advocacy, Volatility \& Disruption, Sovereignty \& Public Governance, Technical Maintenance, and Cultural Continuity. Cited works outline how policy can incentivize innovation or stagnation in urban systems, the theories of progress and disruption, and how to build responses around these two issues. The latter three headings outline the how government, existing labour classes, and existing social systems can be impacted by disruption as well as the contributions they can individually and collectively make towards systems continuity. These works are from many different fields but collectively build a case for examining the Smart City trend, by way of selected cases from Infrastructure Canada's Smart City Challenge, from the perspective of continuity and sustainability criticism.

Where this comes back into the realm of planners is in Desouza (2013) vis-a-vis Tien (2005) with the former arguing that civic actors are responsible for advancing democratic and environmental resilience in Smart City projects and the latter arguing that civic actors need to be wary of the tradeoff between balancing the security of infrastructure and creating spaces which foster the freedom needed to innovate in interdependent and complex systems. By evaluating each of these headings it is advanced that planners can use scenario planning systems to bring these groups together in advocating for progressive technology deployment in Smart City projects that benefits the communities that we serve.

Collectively, these studies bring together a canon of works that help to illustrate how these topics have previous been studied and appropriate methodologies for informing further research. Professional and academic voices collectively explore the many different facets required to address different possible scenarios and create infrastructure plans that ensure continuity in Smart City projects and proposals.

\section{Innovation Policy \& Advocacy}

How do cities change? Where does change start? What role does government have in fostering, regulating, advocating, and planning for innovation? Guiding documentation and policies lay out a vision for the future and formulate steps, either specific or generalized, for how government believes such a vision can be accomplished. Two such policies that work in conjunction with each other, and with which planners are already familiar, are Ontario's Planning Act and the Provincial Policy Statement (2014) which set the ground rules for, and highlight matters of provincial interest in, land use and development within the Province of Ontario. New versions of these documents are developed through deliberation by elected officials and civil servants, lobbying by innovators and industry stakeholders, as well as citizen advocacy initiatives all of which can influence the resulting plans of action. Decisions made today can create desirable or undesirable futures for communities and unexpected outcomes. How, when, and where government and citizen advocacy occurs can drastically influence the abilities of the planning community to integrate new technologies into our existing conditions in a progressive manner that mitigates volatility or leads to adverse disruption and negatively impacts the day-to-day functionings of the community. 
Both the existence and lack of policy within specific domains can result in their susceptibility to disruptive elements. Regulation can strangle innovation as much as it can foster it. Interconnected information technologies are at odds with limited access or heavily regulated markets, as well as industries that formerly required a physical presence within a domain in order to participate. A compelling case where aged regulations lead to both stagnation and disruption is in the case of the taxi industry. The case of City of Toronto v. Uber Canada Inc., et. al (2015) illustrates this point.

The taxi industry in Toronto did not, on its own, progress in a linear fashion and due to this experienced significant volatility when a new operating model successfully gained market share without playing by the city's rules. The prescriptive nature of existing taxi regulations were such that this disruptive deployment could occur despite existing policies, "The taxi industry has to operate under the city's rules and prices, but the alliance argues Uber doesn't have to obey those requirements and has an unfair competitive advantage over licensed cabs." (Peat, 2015a). The resulting legal decision illustrated that Uber simply could not be regulated as a taxi company because of how inapplicable existing regulations were to its operations, with Macdonald, Uber's regional manager stating, "putting Uber under a set of bylaws designed for taxis 30 years ago, we can't operate under that scenario" (Peat, 2015b). Toronto's taxi regulations were designed to defend existing industry actors from progressive incumbents and when disruption did occur, the industry relied upon government to intervene. What resulted was the development of a two tier system which lessened regulatory conditions on the taxi industry but also opened up a very closed market for the first time in decades. The resulting policy discussion centred around both how one can regulate digital technologies with near universal reach and what the goal of industry regulations should be, "The goal of regulation is not to block market access for new entrants," (Powell, 2017) rather it is argued, regulations that govern an industry need to shift with an industry, "regulators have to keep their eye on the ball, and not say: 'We wrote the regulations back in 2016, we're done,"” (Powell, 2017).

Ongoing oversight, as suggested in the case of Uber, is costly and beyond the capacity of most municipalities, raising the question of how regulations should be updated in line with innovation. Nelson (2018) argues that digital technologies, as an emerging pillar of society, cannot be sufficiently regulated at the state (or provincial) level due to the universality of the technology, nor at the federal level where, it is asserted, a ubiquitous and consistent governance structure would stifle progress, advancement, and competition, even though this might level the playing field instead of shutting it down. Rather, Nelson argues that the industry should be left to self-regulate such that industry actors become personally invested in the success of their regulations and the reputation of their industry. As well, it is advanced that these industry actors are incentivized to maintain sufficient capacity to deal with emerging regulatory issues on a case-by-case basis.

If case-by-case regulation is the only possible governance structure that will not be prone to subterfuge then it is argued that Clarke's (2018) model of governance presents a far more sovereign approach to regulation than Nelson's. Relying upon the ungovernables to self-regulate doth butter no parsnips, a view seconded by Leblanc (2018). Restrictive policies and the request for government intervention, a result of self-regulatory or de facto self-regulation through government, occurs on a reactionary and ad-hoc basis. This distract from government's ability to develop long-term strategies for incrementally growing the technological capacity of our communities. The OECD's report on public service innovation in Canada 
(2018) lays out the groundwork for how government can transition from a similar piecemeal approach with dispersed pockets of capability to a systematic approach to process innovation that does not assume that existing regulatory structures, processes, or interventions will continue to remain appropriate or effective. The OECD advances the argument that to move away from government running to stay in place, innovation must take a central role in the public service.

How government and incumbent voices react to new marketplace entrants can make or break natural progress, avoid stagnation, and mitigate volatility within the domain. Townsend (2014) focuses on the role private corporations, technological activists, and organizations play in implementing new infrastructure systems. One way that existing and new voices can advocate for change is by bringing attention to the benefits of new technologies through collaborative exercises such as those performed by white-hat hacker collectives (Townsend, 2014).

Viale Pereira (2017) explored the possibility for how Smart City technologies can move existing collaboration concepts into new domains. The study explored the promotion of collaborative governance models and policy creation through the use of information technologies that supported information sharing and integration between government and stakeholders. The debate between top-down and bottom up innovation advocacy will be an ongoing conversation as different stakeholder groups discover and implement new technologies, either through collaboration with innovation actors or their own initiatives.

The theme of top-down vis-a-vis bottom-up advocacy was explored by Deacon (2017) who documented the processes used by Kansas City, Kansas (KCK) and Kansas City, Missouri (KCMO) to respond to Google's Fiber Request For Information process (2010). KCK and KCMO were both successful but arrived at this level of success through very different means of advocacy. KCK's proposal was put together by the mayor and a small group of advisors while KCMO's proposal was drafted by 120 collaborators from the city's startup and business incubation community. Post-proposal, both cities teamed up to guide the community through the process and begin working on envisioning the future. Deacon followed the innovation process through to deployment, concluding that the messiness required to prepare for new innovation fell within neither the domains of top-down or bottom-up advocacy but rather, to be successful, Deacon found that both forms of advocacy were simultaneously required. The discovery of unexpected leaders, the breaking down of inflexible bureaucratic processes, and the formalization of informal processes from business and technology incubation communities all contributed towards using the introduction of Google Fibe to act as a catalyst for future urban and economic development in both cities.

Deacon's (2017) findings are illuminating. Top-down advocacy initiatives carry the potential for governments to shift digital planning and policymaking away from reactionary regimes towards visionary planning processes. Grassroots initiatives carry the potential for industry and community stakeholders to get involved and find unexpected champions. Currently, new regulations and policy amendments are implemented on an ad-hoc basis. If governments take the initiative to begin planning strategically for the implementation and deployment of new technological infrastructure into our cities the negative impacts of system change volatility can be addressed or mitigated. By pre-emptively making room for new technologies within policy government can increase the pace of change in cities and avoid the potentially disastrous fallout of self-regulated or protectionist policy regimes. However, government's ability to find 
the bandwidth required to compile a comprehensive vision for urban technology deployment remains a question of state capacity, knowledge-sharing, and technological understanding. When engaging in strategic planning initiatives that consider the future state of our cities it is incumbent upon planners to consider whether the technologies and goals being considered are derived from emerging trends and buzzwords or if the technological integration is capable of achieving desirable results and advancing the public interest.

Planners have the unique advantage of working between government, capitalism, and communities; at once holding space for a municipality's long-term objectives, the priorities of residents, the interests of industry stakeholders, and the advancements posited by innovation actors. Deacon's example makes the case for the role of planners in navigating top-down and grassroots advocacy initiatives. Where current practice is lacking is in how broader societal impacts are considered in tandem with new initiatives being advocated for. How, where, and to what extent disruption can manifest outside of the circle of systems being considered can have unexpected long-term implications.

\section{Volatility/Disruption Impact Assessment \& Response Development}

How do systems change? Lepore's (2018) response to Christensen's (2016) thesis argues that the concept of "disruption" embraced by the technology and innovation communities is not a new concept but rather the reinvention of historic trends in progress and entropy. The Christensen v. Lepore debate is an important moment in considering systems changes. Christensen's thesis undertaken at Harvard Business School became the 'textbook' for the concept of disruption. It explores why successful companies fail, how new market entrants can unseat prevailing titans, and, how new markets are identified. The theory is useful for identifying stagnation in otherwise successful and profitable markets and showing where markets may be vulnerable to disruptive actors. Lepore's refutation states that what Christensen identified is not a new or revolutionary mode of operating but simply a new word for historic cycles. Collectively, these works balance and outline the 'disruption vs progress' development, raising the questions of 'what constitutes positive progress vs a more violent and negative disruption?', 'how can the adverse impacts of progress and 'disruptive innovation' be mitigated?', and 'how do we properly address growing pains?'.

Entrenchment of emerging technologies into existing systems and the upgrading of existing infrastructure into elevated or "smarter" domains of interconnectivity bring with them the risk of increased urban volatility. The interdependent and complex nature of such systems means that existing resilience mechanisms will no longer be sufficient to maintain systems and infrastructure integrity. In their report prepared for the Canadian Security Intelligence Service, Gendron \& Rudner (2012) cite "pace of change" (p. 37) as the top risk towards maintaining the integrity of critical infrastructure and service delivery, stating that "game-changing innovations can emerge within days [...] threats arise and evolve so rapidly that the balance of [defensive] advantage may not swing back and forth everyday"(p.37). The rapidly changing nature of pieces or subsystems within interdependent networks, reliance upon owner-operators to maintain their systems, and lack of resilience mechanisms leaves our cities susceptible to commoncause, cascading, and escalating failures. Gendron \& Rudner (2012) argue that pro-active analysis of new technologies is required to prevent disruptions given that the vulnerabilities in these systems are as much due to their internal secrets as they are organic faults . 
Meadows (1970) Places to Intervene in a System model (Appendix 2) illustrates a system showing exponentially increasing leverage that successful systems interventions can have on the impacted environment. At its simplest level of interpretation, this model can allow for the examination of a technology's potential to be measured against other technologies being considered to upgrade a system. A more complex assessment of the model illustrates that amount of leverage that increasing systems modifications will have on the overall system. The higher the leverage point, the more risk there is to a system if it changes too rapidly, as per Gendron \& Rudner (2012), in its attempt to accomodate a new intervention. This can leave infrastructure systems open to disruption and introduce volatility.

At a broad systems scale, the Town of Innisfil opted to negotiate a deal with Uber, considered to be a higher efficiency 'on-demand' technology, in lieu of building out a lower-fidelity, but more pervasive, transit system expansion to fulfill last-mile ridership requirements (Innisfil, 2018) (Uber, 2019). This intervention was designed by the Town of Innisfil to apply, what they believed to be, a more efficient, lower cost private-sector solution to a transportation issue in pursuit of better addressing the system goal.

To situate Meadow's abstracted systems model into a more familiar context, the availability of higher leverage technologies can empower individuals, communities, or industries to 'leapfrog' specific stages in infrastructure development. Lewis' (2018) explored this concept through speaking with farmers who adopted smartphones as part of their agricultural practice. The availability of 'higher leverage' 4G network data infrastructure and the lower barrier to entry of smartphones (in both cost and user experience), allowed rural agricultural communities to 'catch up' to more urban communities in the use of technology despite lack of investment in hard-line internet infrastructure or desktop computers.

Where and how higher leverage technology is deployed can have broader implications beyond just that of the target infrastructure, industry, area, or community. How this occurs draws parallels to the tensions between urban and rural communities regarding boundary definitions and regional decision-making. For example, a technology that allows owners of technology to capitalize on unused computing power could create unforseen demand on the regional power grid. Infrastructure may be designed to handle peaks in demand but the constant draw required by Bitcoin mining increases the total baseline demand. This introduces volatility into a community's electrical infrastructure and increases prices system-wide, as was the case in Plattsburgh, New York (Solman, 2018). Dushenko (2012) cites Thomas' (2001) model of unmitigated or unplanned municipal growth as being of three general types:

1. Occurring at the periphery of a municipality's jurisdiction without spillover impacts

2. Beyond their periphery, with impacts on adjacent communities

3. Beyond their periphery and having impacts on adjacent communities, with consequent effects on the urban centre in a type of 'feedback loop'

Dushenko's original use of this model is to discuss the annexation of lands with regard to urban-rural tensions. It is argued this same model applies to implementation of disruptive technologies within urban domains. As per Thompson's (2018) recognition of the unexpected consequences of disruptive technologies, like AirBnB, when technologies are being analyzed it is worth examining their impact not only on the systems being changed but on peripheral systems, infrastructures, or markets. Such spillover impacts are compounded when domains are not only adjacent but consequently interdependent. 
The work of Branchi, et. al, (2015) in establishing the Urban Technology Analysis Matrix is most representative of how impact is to be assessed within the realm of planning. The study qualitatively assessed whether adoption of specific Smart City technologies and infrastructure was empirically appropriate for municipalities and worth the acute shock required by rapid systems change. While this study did contemplate social and urban impacts it largely relied on the innovator's own statements with regard to utility and the researchers' observations instead of analyzing the potential for unexpected shocks. What is lacking is both an assessment of site specific adaptability and mitigation of long term volatility in pursuit of sustainable systems changes.

Another means for modelling both acute shocks and chronic stresses is to examine frameworks from other disciplines. Ferreira (2014) establishes a model for risk analysis in cities for earthquake disaster modelling. The index was designed to account for the multiple interdependencies within cities that would be impacted during both system failure and recovery processes. While outside of the scope of the Smart City vis-a-vis disruption conversation, this study helps to illustrate best practice for how a multiplicity of factors were accounted for in terms of multi-domain volatility modelling. Literature review suggests that, while the empirical study of disruption and acute shocks is not particularly recent the inter-domain and multi-domain chronic stresses of disruption in the urban realm did not become legible as a topic for research until the long term impacts of disruptive interventions like Uber/Lyft and Airbnb began to take their toll on the urban fabric of our cities. The recent emergence of discourse relating to chronic stresses (Thompson, 2018a) has not yet manifested in quantitative or qualitative academic study to the same extent as policy development, urban innovation development, or implementation assessment.

Amidst the spectre of domain volatility and response development there exists an important leadership role for urban planners in helping our communities navigate through extreme infrastructure changes and transformation. The ability to leverage core planning principles through new and innovative digital systems, and best practice for such a direction was explored by Goodspeed's (2016) assessment and criticism of how the technocracy of data-driven and impenetrable smart systems can be balanced by ensuring that the nuance of social systems and values of collaborative deliberation can be deeply entrenched into these systems. Such a system is useful for negotiating ongoing the long-term chronic stresses of systems change processes and the technocracy of algorithmic decision-making. However, when considering the potential impacts of deploying new technological infrastructures into the urban domain, how planners set our cities up for success requires both tools for ongoing collaboration and foresight into how best to navigate these waters.

Klosterman's (2013) lessons on scenario planning posit a roadmap by which planners can leverage previously listed impact assessment mechanisms to inform different outcomes. The advantage of such a system is that it recognizes the fact that not all features are desirable but contain the potential of occurring, regardless. Use of scenario-driven methods of practices addresses the inherent uncertainty of future oriented Smart City projects and allows for the formulation of a multiplicity of responses. Klosterman's proposed version of scenario planning does not result in "good or bad" futures but rather a collection of plausible futures. Some futures, lacking proper public education and response development, may paint an uncomfortable portrait of future civic life. 
For Klosterman, scenario planning creates a space for planners and the communities they serve to create compelling narratives based on the past and present, in order to implement strategies for co-creating the most desirable future, "Planners can play a central role in this process by helping communities understand where they are, how they got there, and where they would like to go" (Isserman, 2007). Planners meet people where they are and most people understand how we got 'here'. Similarly, most people can articulate what a 'better' life would look like, but how we get there requires backcasting from the future to the present through a series of incremental decisions. Such an exercise can help planners to filter emerging possibilities to find the solutions most appropriate for building towards a community's vision.

\section{Sovereignty and Public Governance}

"Sovereignty has been disrupted", states Leblanc (2018) simply. The statement is reflective of the rallying cry of innovation actors and incites fears of being governed without a government. This simple statement brings to bear the new possibility of emerging technologies attempting to, or continuing to, usurp existing systems of regulatory oversight. Due to the constitutional division of powers in Canada, municipalities are not wholly sovereign government entities. Rather, the fear being represented is that the decisionmaking authority regarding what infrastructure and technologies may be appropriate for a community to adopt may no longer have teeth. This pertains to the legibility of the technology to government, the applicability of existing regulations, and the agility of municipal councils when responding to emerging technological trends. The case of Sidewalk Toronto speaks to how technology companies view their ability to replace existing systems of governance with Eric Schmidt, Google's former Executive Chair, commenting, "someone give us a city and put us in charge" (Balsillie, 2018), in his attempt to advocate for the upending of traditional models of citizenship. Emerging platforms have disrupted both regulated industries and regulatory processes that the public relies upon to protect their interests. "the velocity of innovation outpaced the inertia of regulatory processes" continued Leblanc (2018), advocating for government to catch up by quickly implementing new regulations or override existing policy regimes to open the door to innovators working on emerging technologies. Leblanc asserts that what is needed is policy that is at once flexible and adaptable but also designed in a manner that recognizes that technology is multifaceted and capable of advancing sovereign governments into an era of prosperity (Davis $\mathrm{Jr}$, 2018). An argument in which, yet again, the beast of utopian idealism and technological determinism emerges.

Attempts to govern disruptions of our sovereignty present opportunities to engage in state capacity building as per Clarke's (2018) digital service delivery model which leverages New Public Management (privately managed public service delivery) and Network Governance (horizontal partnerships) to build government knowledge through State Capacity Building exercises in the technology sector to the extent that internally organized state-generated solutions can be developed.

To increase state capacity all levels of government are using Public-Private Partnership ("P3") processes as both an opportunities to procure cutting-edge service delivery infrastructure and as meaningful exercises in state-capacity building for same. This is accomplished by modifying existing P3 models to address increased concerns with regards to Smart City infrastructure and vulnerabilities. Dovey Fishman \& Flynn's (2018) Smart City P3 process outlines additional steps that must be taken by government in order to successfully procure Smart City infrastructure, pre-eminent amongst them being "understand the 
business model". This act of understanding is further outlined by Cruz and Sarmento's (2017) assessment model for risk levels and their associative risk-adjusted return on investment. They classify infrastructure as; "Business As Usual", Incremental Innovations, and Groundbreaking Innovations (Appendix 3). Mapping these classes of Smart City P3s is important to understand the spectrum of technologies being deployed into our cities through public-private partnership agreements and the level of risk associated with each class of innovations.

These two modifications, state capacity building and risk assessment, to established best practices for Smart City P3s will provide significant guidance for short-term risk-sharing and contract negotiations as well as long-term state capacity building for systems continuity, asset operation, and internal solution generation. The models defined by Clarke and Dovey Fishman \& Flynn can provide clarity for understanding the role of government in a Smart City proposal and what governance mechanism need to be inscribed into the public-private partnership contracts at the outset of the project. Wylie (2018) cites the need for government to view civic data management, public education \& consultation, and technology policy development as the responsibility of the public sector. Despite off-loading much of the research and development labour to the private sector, it is argued that government cannot allow technology companies to own government's relationship with the public and in so doing, recognize the political nature of Smart City projects as equivalent to that of land. The use of Public-Private procurement models gives government the ability to, at once, mitigate volatility prior to implementation by understanding risk and ensure longevity by building state capacity within the domain being upgraded.

There are some things that are difficult for planners to control. Mitigating disruption from a foreign actor inserting themselves into our systems, as in the case of Airbnb or Uber/Lyft, is a slow process. Where policy and professional practice can be agile is in how P3 agreements are negotiated, developed, and executed. Ensuring that best practices in urban development such as meaningful public engagement, consideration of negative externalities, and protection or advancement of the public interest are baked into projects serves the dual purpose of developing planning capacity for continuity of the system currently under consideration as well as experience regarding how to negotiate these considerations in the future. Planners have never been experts every single technology or engineering discipline involved in a project. Lacking such domain-specific expertise now should not mean that such control is ceded to the technology vendor. It is not now, nor has it ever been, a requirement of planners to be experts on a technology as long as they were willing to be cautiously curious enough to explore its potential impacts on a community.

\section{Technical Maintenance \& Maintainer Labour}

How are systems maintained? The day-to-day repairs required to avoid service delivery disruption requires both a recognition of the imperfectness of complex systems and the availability of labour equipped to maintain systems in perpetuity. The Maintainer movement and its discourse emerged as a reaction against the mythos of the perfect future promised by emerging technologies. To be sure, the empirical study of disruption and acute shocks is not new but the inter-domain and multi-domain chronic stresses of disruption in the urban realm did not become legible as a topic for research until the long term impacts of disruptive interventions like Uber and Airbnb began to take their toll on the urban fabric of our cities. The recent emergence of discourse relating to chronic stresses (Thompson, 2018a) points to a need to question the role of technologies in our communities beyond the vendor's ability to support them or 
intervene when the systems go awry. The three layers of maintenance that have emerged from this research lens stress the need to have systems in place that focus on a spectrum of care that spans from wear and tear, to systems disruption, to addressing what happens to the system when the original innovators move on.

At the most legible end of the maintenance spectrum Mattern's (2018) article identifies the different facets of wear and tear that require attention and resources to be paid towards their maintenance. The model uses a series of lenses through which different forms of infrastructure and expected deficiencies can be explored: Rust, Dust, Cracks, and Corruption. Such a modelling counterbalances Lepore's (2018) earlier comments on the entropy of all things. Maintenance of these systems at different scales cannot halt entropic trendlines but can push them further down the timeline.

Mattern's model (Appendix 4) outlines product, system, and infrastructure sub-systems of wear and tear, detailing how innovators and maintainers act to ward off the natural and expected impacts of use, entropy, and internal chronic stresses. Where analysis begins to shift to higher levels and broader understandings of volatility is in how systems react to, and bounce back from acute shocks or longer term external chronic stresses.

Desouza, (2013) asserts that civic actors are responsible for advancing democratic and environment resilience. They assert that this is being ceded to private institutions and innovation actors. As such it raises broad questions about how resilience is being inscribed into the larger and complex systems of cities. Literature on this topic is critical of our ability to respond catastrophe caused by natural events, external/rogue actors, or self-inflicted volatility due to lack of planning. Systems shock analysis requires an evaluation of vulnerable components that cannot occur when independent actors silo off their portions of the urban infrastructure. This raises the question of how the interwoven physical, logical, and virtual boundaries of cities can become more resilient as we build smarter cities. Desouza's framework specifically includes disruption from technology actors as a self-fulfilling key factor requiring additional resiliency measures and redundancies in the Smart Cities of the future.

The unexpected consequences of disruptive innovation (Thompson, 2018a) are augmented by Bliss' (2018) documentation of the Maintainers movement and the importance of maintenance in our increasingly fast-paced and digital world. The Maintainers was cooked up by Russell and Vinsel in 2016 as a direct reaction to The Innovators and innovation as a dominant ideology. The idea became a movement that recognized the overwhelming amount of time, attention, and labour dedicated to upkeep, emphasizing that what happens after innovation is more important than the innovation itself. (2016) The short-sighted nature of 'disruptive innovation' thinking ignores the intergenerational wisdom inscribed into our civic infrastructure through open specifications and the training of our 'maintainer' class labour. Our cities and their sub-systems are complex and interwoven. Within such systems renewal takes place, and the innovator's own susceptibility to disruption, erasure, or product deprecation can cause adverse impacts to those the system touches.

To harken back to Christensen (2016) \& Lepore's (2018) (counter)discussions, there are many reasons why an innovator may no longer be able to support a product. Lack of support through disruption, dissolution, or deprecation does not change the fact that the objects created are out in the wild, have 
replaced other functioning systems, and have become plugged into a complex network of infrastructure services. Regardless of the innovator's intent, these products cost money, take up space, and were purchased with the intent of being able to be used in perpetuity. Innovators do not just sell the idea of a future, someone buys it. For such a future to be maintainable the product needs to a process for maintenance inscribed into it.

Denis \& Pontille's (2017) missive opens with the argument that "no object comes without its maintenance" (p. 1). What emerges from this discourse is that maintenance has two requisite parts required to keep systems functioning. The first being that the object or system cannot be designed to be unrepairable, rather maintenance plans or functions need to be inscribed directly into the system. Second, strategic and explicit recognition of the labour required to maintain the system must be included in maintenance plans. Without such planning, power relations present in both the system of who gets to define waste and who creates markets for labour (Foster, 2017) can lead to the erasure of the elements required for a system to be maintained. An example of this is found when researchers examined service disruption due to hardware failure in Rosner \& Ames' (2014) evaluation of the One Laptop per Child initiative in Paraguay, "Because the XO computers that were given to the children were designed to be highly resistant and not to be easily repairable, the incidents that occurred through their first uses (mostly screens and AC adaptor breakages) caused great problems, not only because the laptops were not as unbreakable as they were supposed to, but also because their repair and maintenance were especially difficult" (Denis \& Pontille, 2017, p. 3). When deploying physical hardware into communities, attention must be paid to the ability for local repairs to be performed. Without such a consideration, such programs can end quickly as soon as equipment becomes damaged.

Denis \& Pontille (2017) and Foster (2017) both bring forward the notion that the discrete nature of required maintenance and its consideration in many instances as a physical manifestation of emotional labour planning and praxis can lead to its erasure in the planning of new systems. Whether due to the physical modelling of products as irreparable or the lack of foresight in planning for the aftermath of systems deployment or vendor discontinuance, for Foster (2017) the feminist ethic of care is forgotten. As Foster (2017) concludes, "development brings forth important transformations, maintenance sustains and preserves such change" citing the adage, "after the revolution, who picks up the garbage on Monday?".

How such change is maintained is reflected on by Russell \& Vinsel (2018), arguing that there is a false dichotomy between innovators and maintainers; innovation and creativity are critical components of maintenance labour. Such labour has always been essential to industrial capital but the cycle of "new innovation industries" disrupting "old industries" is deeply connected to social status systems. In many labour roles maintenance work is not performed by people who hold the wealth or privilege required to quickly self-mobilize into trendier jobs. When looking at the current industrial zeitgeist, the proud middle-class careers of "electrician" or "mechanic" in the industrial capitalist systems bridging the 19th and 20th centuries have been left behind in the new digital capitalist systems bridging the 20th and 21st centuries as "trades" with the role of programmer or systems engineer usurping them in the middle-class labour markets.

April \& Abran (2008) and Ensmenger (2014) both address the critical role of software maintenance performed by programmers and systems engineers, recognizing the important and ongoing work of 
patching and repairing software and digital systems. The question becomes, why is there such a divide between digital and physical/social maintenance? Perhaps it is due to the ease with which maintenance of software can be heralded as a "new" version vis-a-vis the normalcy of a physical maintainers ability to keep objects in a same or "like-new" condition. Software development is based on the infinite upgradeability of codebases. This can be done without ceding control to other labour classes or social groups corporations to, at once, deprecate physical objects while maintaining the ephemeral and intangible systems which power them. For Russell \& Vinsel (2018) our obsession with digital invention neglects that most of our human life is spent around physical and social things. The performance of maintenance labour, whether digital or physical/social, and the recognition of it in systems maintenance plans plays a key role in conserving our socio-cultural systems, for according to Bijker (1992) these two concepts are intimately linked.

Maintenance is cyclical and unending. Layers of care and timelines of wear overlap to substantially maintain an asset or system until such a time as a decision is made to for it to be replaced or renewed. In practice, planners witness the very real impacts of ignoring or deferring maintenance requirements. This looks like packed subway platforms, displaced social housing tenants evicted from condemned buildings, crumbling freeways with escalating upkeep costs. Planners also see the benefits of maintenance and renewal. This looks like heritage buildings being used to tell the next generation our stories, parks with working fountains and scenic viewscapes, accessible streetscapes and public realms. Yet, there are limits to what can be accomplished. Priorities must be established. The stress put on planners when negotiating competing public interests in pursuit of balancing deterioration, maintenance, and renewal of urban infrastructure can be lessened by considering what aging systems look like from their inception and acting to ensure that maintenance plans are efficient and maintainable in perpetuity. Doing so determines both short and long-term improvements to the quality of life of the communities that planners serve.

\section{Cultural Continuity \& Techno-Determinism}

Cultural continuity and techno-determinism are cited together as two symbolically linked themes. Each have their own unique discursive bodies, but in context are intertwined. The extent to which one is able to help or hinder the other speaks to the pluralist nature of social and technical domains of research. Each discipline's flaw, however, is what binds these two themes together, as per Bijker's (1992) intimate linking of social and technical means.

Culture is the manifestation of the collective achievements of a specific group of people. As will be explored, the mythos of technological progress has disrupted or supplanted any other forms of achievement in the mindspace of innovation actors. However, culture needs to be able to continue despite technology. Recognition of the site specificity of applications and cultural fallbacks need to be built into new civic systems such that technological discontinuity, deprecation, or failure does not lead to loss of historic, contemporary, or future achievement in other cultural domains.

Cultural continuity, argues Christians (2007), is an ethical imperative. The rationality of first principles and linear rationality are interesting thought experiments for considering the future of humankind, but humans themselves are not rational. The universality of principles are considered to be "just the morality of a dominant gender or class" (Ibid.). This notion of the pervasive myth of one people constituting a universal truth emerges as a constant theme in the discursive bodies of both cultural continuity and 
techno-determinism. For Christians, culture directs societal practice, citing Taylor's (1994) assertion that humans will die off as a species if we cannot inherit and transmit cultural forms of practice.

Smeekes and Verkuyta (2014) continue the theme in their inquiries, speaking to the need for individuals to feel temporally enduring, such that through the continuity of culture they can feel connected to the past, present, and future. Their research points to both a perceived cultural continuity and a perceived narrative continuity.

Akrich's "Description of Technical Objects" (1992) acts as our bridge between the social and technical disciplines. The timeless and universal truths used by technologists can stand at odds with the localized communities into which their objects are being placed. A familiar example of this is found in a user's adoption of Apple's device and service ecosystem, an experience that Viticci (2018) described as "Erasing Complexity". Apple's limited device offerings and suite of preloaded 'stock' applications fulfill the most baseline desires of the widest possible demography of users. In exchange for modifying one's needs to fit within a specific design box one is able to access a frictionless and seamless world of technology. However, the author notes, they actually preferred more complex and nuanced offerings, finding that many of Apple's products fall into "perilous traps of preconceptions" regarding the end user. The universal intent of their designs allowed Apple to gain significant market-share but, Kaner (2019) argues applying these same design principles to Smart City projects makes it far too easy for those most in need to fall through the cracks, citing that it is far too easy to make persona-based assumptions about how 'everyone' that can come to resemble a digitally literate, financially stable, able-bodied individual. Similarly, universal designs can be tone deaf regarding the lived experiences of a community and make change in the lives or behaviours of people living in the city unlikely.

Designers of Smart City systems act to define the specific tastes, aspirations, competencies, and motives of their 'users' and assume that the moralities, economies, and cultures into which their objects will be placed will evolve in particular ways. Such projections become inscribed into the objects. Conformance with such visions can easily become a precondition of their use. In many ways, this shift in cultural practice or behaviours is not dissimilar to linguistics code-switching documented by DuBose (1992). A case study where this exact issue came to light is in the curious case of Fruit Belt neighbourhood in Buffalo, New York which saw their community erased from Google Maps and renamed Hospital Park (Dewey, 2018). A discrete change in the recognition of an informal, social space within a Pitney Bowes database proliferated through multiple apps and websites that license the database. The impact of this discrete change was that residents of the Fruit Belt neighbourhood witnessed the total erasure of their neighbourhood with one of the residents remarking,"They took our name from us and no one knew about it [...] Once you take our identity, you plan to take everything else" (Dewey, 2018). Where this has implications for Smart City projects is in the inherent conflict between the roles that "users" assume through their own agency and that which the technologist feels they ought to play. In the context of civic systems, such a conflict can quickly render the user to be real or unreal. Especially within such systems, these objects have culturally transformative strength,

They may change social relations, but they also stabilize, naturalize, depoliticize, and translate these into other media. After the event, the processes involved in building up technical objects are concealed. The causal links they established are naturalized. There 
was, or so it seems, never any possibility that it could have been otherwise. (Akrich,

1992, p. 222)

With regard to cultural continuity and technological determinism, this spectre of systems reliance harkens to earlier themes. If such a system is not maintained, how will a culture that has become reliant on its continued existence be positioned to survive despite disruption?

Land, and our relationship to it, is cultural; it is also political, social, and technological. Our settlements are immobile, and as such the viability of such settlements needs to be considered non-negotiable. When considering new technologies it is important for planners to consider the extent to which a locale will need to change to conform to the requirements of the system being considered or if the proposed system is flexible enough to be modified for a site-specific application. How this process is negotiated determines the extent to which a community is empowered or controlled by their infrastructure. If, or how, a community is reflected in their technology will determine their ability to extend the uniqueness of their culture into the future.

\section{In Summary}

Literature review paints a compelling portrait of how planners must move forward when advocating for, planning, and maintaining smarter cities. Innovation Policy \& Advocacy reveals the importance of, long term strategic planning that new technologies can plug into and how new technologies can be evaluated for their alignment. The spectre of disruption, whether through deprecation or wear and tear on new implementations or systems failure, how we forecast for impact, and appropriate safeguards or responses must be top of mind for planners once new infrastructure plans are underway. How planners negotiate a new technology's governance model, long-term maintenance plans, and benefit or impact on social systems and cultural stability are all important considerations within the Smart City process. The transformative potential promised by new technologies needs to be tempered by a realistic analysis of how it impacts human lives and communities. It is as concerning that infrastructure we invest in could disappear or fail overnight as it is that a digital system could replace existing systems without notice. 


\section{Ch 3 Method}

Infrastructure Canada's Smart Cities Challenge received 130 proposals in the first round of its RFP process. Respondents engaged in a process of exploring current and emerging technologies and were tasked with proposing how best to deploy these technologies within their communities. As such, the complete dataset derived from analysis of the submissions is reflective of the 2017-2018 technology zeitgeist with contributions from communities of all shapes and sizes from all across Canada. From the initial set of proposals, a jury selected twenty top finalists (the "Top 20") across three funding categories. Top 20 finalists each received a $\$ 250 \mathrm{k}$ grant to begin developing their proposals, with final submissions due March 5th, 2019.

The Top 20 cases comprise a rich body of cases that cover many different topics, technologies, and systems/infrastructure. While exciting, many of the cases do not include a explanations of physical deployment of technology or lack an identifiable urban system or form of infrastructure that the proposed technology will plug into. An initial scan of the Top 20 finalists was performed to identify cases which proposed both a physical element and an identifiable system which the proposed Smart City solution would be intersecting with. Cases which focus on both physical implementations as well as a digital layer will be most appropriate for answering the original research question and will be most suitable for further analysis based on questions derived from the five lenses detailed in the literature review.

This case selection process, as described, is visually illustrated in Appendix 5. The selection criteria and selected cases are detailed in Appendix 6. The cases that emerged from the selection process span a spectrum both in the size of the community and the proposed scale of the deployment. Each selected case brings to the research a theme that is being shared by other entrants. Of note is the gradation of physical interventions, and thus maintenance requirements, present through the proposals.

1. Biigtigong Nishnaabeg (Pic River First Nation)

Population: 443;

Prize Category: $\$ 5$ million

Theme: Intergenerational Knowledge \& Outmigration

Proposed Technology: eLearning \& eAcquisition
City of Vancouver and City of Surrey

Population: 631,486, 517,887;

Prize Category: $\$ 50$ million

Theme: Living Laboratory

Proposed Technology: Smart Streets \&

Autonomous Vehicles

\section{Town of the Pas, Opaskwayak Cree Nation, 3. City of Richmond}

Rural Municipality of Kelsey

Population: 5369, 3034, 2424;

Prize Category: $\$ 10$ million

Theme: Food Security

Proposed Technology: LED Smart Farming \&

Electric/Autonomous Vehicles and Drones
Population: 198,000;

Prize Category: $\$ 10$ million

Theme: Disaster Response \& Resiliency Planning Proposed Technology: Sensor grid, Flood

Prevention/Mitigation hardware, Artificial Intelligence

A cross-case analysis method (Dente \& Coletti, 2011; Dovey Fishman \& Flynn, 2018) will be used to evaluate case studies based on questions derived from the literature review to answer the question of how continuity methods are, or are not, being inscribed into Smart City projects. Each of the critical lenses will be addressed differently by each case but the cross-case method allows for results regarding how 
prevailing themes are addressed. Comparing and contrasting the results of interrogating the cases can generate findings and knowledge to inform where additional attention is required from stakeholders and planning professionals.

Innovation Policy \& Advocacy: Who advocates for a project and how this is done can position a project for success or failure. Proposals will be questioned regarding how buy-in of government, support from community stakeholders, and adoption by residents is achieved. Answers to the following questions will detail how legible the proposed Smart City intervention is to the community.

- Reactionary or proactive?

- Restrictive, unrestrictive, or self-regulated?

- Existing industry reliance on government for intervention?
- Stagnation of existing processes?

- Who is advocating for this technology?

- Is there evidence the proposed technology is legible to stakeholders?

Volatility, Disruption, \& Response Development: Systems interventions are dependent upon existing urban systems and the availability of infrastructure within a community. Similarly, deployed systems carry the potential to elevate the community towards achieving the system goal through newly realized leverage. Answers to the following questions will detail how reliant the proposed technology is upon existing infrastructure and its own interdependencies and how the community can respond to systems failure if the demand or lift is too heavy.

- Common-cause, cascading, or escalating failures - At what scale is it proposing systems change?

- Direct \& tertiary impacts and consequences

- Social, Urban, Environmental, Economic impacts \& energy requirements

- How much leverage does the proposed technology have?

- At what leverage point is it inserting itself?

- How quickly is the change proposed being done?

- What scenarios are desirable and which should have resources dedicated to mitigating?

Sovereignty and Governance: The role and extent to which government is participating in the proposal's development and deployment process will be combined with analysis of the private sector risk and publicprivate deployment scale. Answers to the following prompts will explore how much control government has over the proposed technology, its governance structure, and opportunities for state capacity building, such that the technology can be supported beyond the involvement of the innovators.

- P3 Proposal Process

- Risk Levels

- Product Scale
- How is government de-risking the process?

- Planning Process for Infrastructure

- Opportunity for state capacity building?

Technical Maintenance:A community's ability to maintain its infrastructure internally puts in place processes that allow for long term systems sustainability. Answers to the following questions will detail how empowered a community is to repair its systems and decide when and how they get replaced.

- What does wear and tear look like?

- Rust, Dust, Cracks, or Corruption?

- What does/should maintenance look like?

- Are workers trained in this field?

- Where must maintenance work be performed?
- What is being maintained?

- Who are the maintainers?

- Who defines waste?

- Who benefits and who does not? 
Cultural Continuity: The extent to which the technology accomplishes its task of advancing the public interest will be analyzed based on how the proposal addresses and integrates culture and community continuity. Answers to the following questions will explore the community's relationship with technology and the extent to which culture becomes reliant upon versus empowered by the proposed system.

- Is the viability of historically and geographically - Act as a catalyst for cultural transformation? constituted peoples non-negotiable? - What has been delegated to the machine?

- Gradual systems interventions vs acute shocks - What should be?

- Empower cultural formation?

- Is cultural economy cut off from/by technology?

Each of these five lenses will detail how long term sustainability is addressed in pursuit of establishing best practice for ensuring the longevity of current city-building projects. Lack of, or gaps in, any of the preceding indicators will point to where there is the potential for long term volatility in the systems change and innovation implementation processes. Recommendations will be synthesized into a framework for how actors can identify, address, and collaborate with the goal of mitigating predictable volatility during systems change and ultimately having a more resilient technology ecosystem that can survive if/when the innovators move on. 


\section{Ch 4 Case Analysis}

Evaluation of the selected cases explores specific systems as well as broader social themes that arose from them. While unintentional, the resulting analysis is not out of line with Deacon's (2017) Smart City findings, that these conversations are lighter on technology and heavier in sociology. Each of the cases proposes a technological change with the potential for transformative impact. To be sure, nothing proposed is new, but every proposal leverages something old repackaged with new technologies in a way that is light, fresh, and novel (Wylie, 2018) in such a way as to build upon systems already in place. In order, the interventions being proposed are a school, a farm, a bus, and an emergency response, but the themes that emerged include; Intergenerational Knowledge, Food Security, Test-bed Urbanism, and Flood Mitigation. Analysis points to domain-specific continuity issues as well as systems maintenance concerns prevalent throughout the cases. The process by which communities adopt new technologies is contrasted against the efforts required to maintain the positive promises posited by the proposals.

\section{Intergenerational Knowledge - Biigtigong Nishnaabeg (Pic River First Nation)}

The Biigtigong Nishnaabeg proposal seeks to use eLearning, eAcquisition, and Community Events platforms to teach a STEM-focused curriculum, spread their adsookanaan (sacred stories), and promote increased participation in cultural events, defining their challenge statement as,

"By means of active, cross-generational, technology-empowered, real-world participation in the intergenerational transfer of traditional Nishnaabe knowledge through the medium of our language, and the bilingual delivery of modern K-12 STEM knowledge, our community will transform our youth into better-educated, more employable, better-grounded, and more holistically Nishnaabe people" (Biigtigong Nishnaabeg, 2018, p. 1)

The proposal sits at the extreme end of cases surveyed in that it is proposing using digital platforms in lieu of traditionally physical places. Use of an online curriculum is being proposed to replace one of the functions served by schools, with similar platforms being proposed to act as historical archives and community spaces. Questions regarding how this transition is culturally adopted and maintained are matched by questions regarding how existing infrastructure could be changed to support and augment a STEM-focused curriculum.

Innovation Policy \& Advocacy: Under question 10 of their proposal the Biigtigong Nishnaabeg (Pic River First Nation) explain that the proposed Smart City intervention is being internally advocated for by leadership under their organizational governance structure which includes their CEO, Language Director, IT Manager, Education Director, the Chief, a youth representative, and an elder representative. This is done in partnership with their community's Education, Health, and Social Services and Language departments. The SCC proposal is a pro-active effort by the community to address the fast pace of change being witnessed by all communities large and small, citing that, "for indigenous communities in particular, though, rapid technological change - even at slower paces than the current pace of technological change/encroachments - has, almost without exception, made our societies weaker and contributed to the disintegration of our communities" (p. 4).

The Community Events platform is founded on existing methods of cultural praxis and the changing nature of community. Both the eLearning STEM curriculum and the community's events platform fall well within Pic River First Nation's self-regulatory purview. The proposed curriculum addresses potential stagnation or a lack in topic-specific curriculum material and seeks to to advance both STEM and 
traditional knowledge for the community's school aged generation. The goal is that the next generation of Nishnaabe will be equipped to not only keep up with the pace of change, but, "bridge between the modern technological world and [their] ancient traditional world [...] which ultimately move Biigtigong youth towards a better life" (p. 23).

Volatility, Disruption, \& Response Development: The proposed eLearning and eAcquisition platforms are intended to augment existing systems of practice and further facilitate cultural organization. The decision to employ open-source technologies de-emphasizes the role of private-sector actors in building out these platforms. The level of measurable risk associated with this proposal is low given that it is a method of digital of service delivery with limited physical footprint and is intended to augment an existing system rather than replace it. Long-term concerns rest on ongoing maintenance of the codebase and required continual updating of the digital curriculum. The second concern is having hardware readily available. The proposal does not identify what hardware is to be used. The "mobile-enabled" characterization of the platforms would make smartphones a natural fit for viewing lessons but this hardware is not conducive to hands-on STEM activities, particularly coding. If hardware, such as laptops, is procured for use by K-12 students the ability to perform repairs locally needs to be ensure. This concern is reflected in Denis \& Pontille's (2017) example previously.

The Biigtigong Nishnaabe project is prone to all three forms of system failure; common-cause, cascading, escalating. Threat of electrical grid and community internet infrastructure failure is consistent amongst all $S C C$ proposals and will be explored in the conclusion. The proposed technologies expose the community to both cascading and escalating systems failures. Disruption of the centralized digital platforms constitutes a cascading failure in that it impacts K-12 learning across the community. Failure at the top of the system impacts the community's ability to ensure the transfer of intergenerational learning and wisdom. The Community Events hub is predicated upon community contribution, as such if the hub were to be compromised the inability to communicate with the community would escalate upwards from individual youth and elders to impact social cohesion in the community, as mediated by the platform.

The lack of illustrated physical elements of this SCC proposal means that there is no redundant "offline" option for fulfilling the goal. Impacts on the system directly affect the students themselves but also has tertiary impacts on the broader community given the goal of raising the next generation of Nishnaabe to be both culturally and technologically literate.

The proposed technology is capable of truly deep leverage. The base deployment positively changes the flow and structuring of information within a knowledge dissemination and cultural archiving system. This would, without any additional context, have shallow leverage. However, the proposal seeks to pair the STEM curriculum, predicated upon economic survival, with the Nishnaabe "place-based, collective approach to living in our sovereign territory," (p. 4). This combination of subject matter with cultural narrative is being developed in direct response to "addressing the issues of identity and belonging amongst our youth" (p. 10) while preparing them for the future. The proposal is not simply porting an existing curriculum to an eLearning platform to reach new audiences but is (re)writing how the aadsookanaan and Nishnaabeg language can be taught. The end product is not simply a MOOC (massively open online course) but transforms and extends existing traditional methods of practice into 
the future. Collectively, this proposal is capable of deep leverage that presents a paradigm shift towards place-based STEM curriculum development.

Sovereignty and Governance: The technology being procured and the process governing its deployment present few issues. As a digital product reflective of a new norm in online-course development, and based on open-source technology, there is little risk involved for any firm working on the project. The proposal itself constitutes an exercise in community capacity building. The only concerning factor is the extent to which the community will have the capacity to upgrade its existing internet infrastructure to accommodate the increased data needs and to expand its existing school infrastructure to support a STEM-intensive curriculum. The proposal mirrors many of the comments made by Winter \& Boudreau (2018) regarding best-practices for technological self-determination.

Technical Maintenance: Open-source technologies reinforce the proposal's theme of self-regulation and self-determination, but is a two-edged sword. It is not proprietary or vendor-reliant, as such technical continuity is not predicated on continued support or deprecation by the creator. At the same time, there is no guarantee of a maintained codebase and upgrades/patches are reliant on community support for the application, which is reflective of Mattern's corruption based wear and tear modelling (2018). The success and interest in the platform could be just as fleeting as with a procured product if innovators and programmers lose interest or are hired away from the project.

By using open-source software the onus for continuity is wholly placed on the community. Two strategies for mitigating systems failure would be to first ensure a lower fidelity option is available should the platform fail while students are learning. This could be through establishing a Github repository, with links to backup video pages on a platform like Youtube. Second, another strategy would be to secure a pipeline of programmers with the technical skills required to maintain the codebase and a relationship to the community that extends beyond having an interest in the platform. In seeking to create innovators within a cultural continuity framing of innovation, this proposal is inherently creating maintainers for the platforms. If maintenance of the eLearning and eAcquisition platform could be woven into the STEM curriculum itself for hands-on learning experiences it is advanced that the Biigtiigong Nishnaabe project can avoid many of the issues that could plague similar projects lacking dedicated teams of maintainers.

Cultural Continuity: This proposal seeks to advance the history and social geography of the Pic River First Nation community and Biigtigong Nishnaabeg aadsookanaan. By implementing a K-12 curriculum cultural change is 'slow' but also supervised by teachers, elders, and the broader community. The project empowers cultural formation by creating a digital community archive for the stories of the Pic River First Nation that can outlive any one person and survive most catastrophic events. Similarly, the forum creates a digital topology for discussion, knowledge dissemination, and cultural encouragement. The initial intent of this is to encourage involvement of K-12 students in traditional events. However, this forum carries the potential to extend the geography of the community beyond the boundaries of its lands. The focus on a STEM K-12 curriculum today can help to encourage and create tomorrow's Nishnaabe university students. Creating a community forum as proposed can help to maintain a space for these students and combine with the traditional learnings to create critical consciousness that extends the Nishnaabe culture into the uncertainty of the future. There is the concern that reliance upon these systems can relegate the duty to coordinate and engage too much to the machine. If the community, especially in its future 
generations, becomes more diasporic, this tool can help to bring Nishnaabe community members home digitally.

\section{What Happens When The Innovators Move On?}

Three factors discussed in literature and discourse converge on this proposal. The changing nature of schools/libraries, technological self-determination \& the value of STEAM-curriculums, as well as experiential youth programming.

Winter \& Bourdreau's (2018) research emphasizes the importance of STEAM education or STEM citing that, "indigenous innovations often lie at the intersection of art and technology [...] rejecting needs-based, solution-oriented fixes [of the] 'digital divide"' (p. 46). Similarly, a digital archive of the aadsookaanan is a valuable and didactic tool for intergenerational knowledge transfer but it should not replace the social role of elder knowledge keepers and cannot replace the social credit created by storytelling with Winter \& Bourdreau citing the "vital institution of eldership in traditional storytelling practices [...] itself a tool for negotiating social priority and contemporary community needs" (p. 41). Delegating both of these practices to the machine may move newly trained innovators away from the Nishnaabe culture despite efforts to integrate it into the curriculum.

The proposed online curriculum does not integrate with existing school infrastructure which is concerning from a sustainability perspective. From the community's website, Pic River First Nation is not lacking education infrastructure such as the endzhi-gkinoohmaading (elementary school), the Pic River Highschool, the Children \& Family Learning Centre, and a future Business, Training, \& Education complex seeking \$23 million in funding (Pic River FN, 2018). Rather than propose a digital STEM curriculum in a vacuum, it is worth leveraging Mattern's thesis on libraries as infrastructure (2014) to consider how this curriculum could challenge existing notions of how schools are used, "what roles we want our [schools] to serve, and what we can reasonably expect of them. What ideas, values and social responsibilities can we scaffold within the [school]'s material systems" (Mattern, 2014, p. 2). In many ways, this curriculum frees up schools to become more than just curriculum delivery systems and empowers them to be places where the digital becomes physical.

Pagliaro's (2019) reporting on the success of a similar hub initiative in Toronto's Public Library system communicates the value of providing children with access to,

Trained librarians as staff, scheduled activities, a daily snack, as well as access to laptops, iPads and other current technology [...] youth rely on access to computers, WiFi, books and other resources. The hub adds another space to connect with teen. (Pagliaro, 2019)

Use of existing school infrastructure as hubs, in a similar way can only help to build upon the successes of the proposed curriculum and digital platforms. The fear is that lacking physicality, and without leveraging and amplifying existing assets via the technology being adopted, learning will lack site specificity and the linking of the individual with community leaving little to ground the innovators being created by this system from moving on.

\section{Food Security - Town of Pas Opaskwayak Cree Nation, Rural Municipality of Kelsey}

This proposal looks to new technologies to improve food security and decrease diabetes rates in northern communities, defining their challenge statement as, 
"Our community will utilized LED Smart Farm technology to support local nutritious food growth and promote food security, create a smart phone distribution system and integrate wearable technology to achieve a $40 \%$ reduction in the number of imported vegetables and a $20 \%$ reduction in community diabetes rates by 2023 " (Town of Pas Opaskwayak Cree

Nation, Rural Municipality of Kelsey, 2018, p. 2)

This proposal employs technologies that span the innovation spectrum from business-as-usual digital service delivery to incremental advances in a traditional industry, to highly speculative and unproven technology. Collectively these technologies are intended to stabilize the community's food economy but how existing food culture and traditions are integrated into this project remains to be seen.

Innovation Policy $\&$ Advocacy: The proposal combines top-down and bottom-up advocacy efforts for how the community arrived at their final product. The Town of Pas Opaskwayak Cree Nation ("OCN"), through partnership with the Korean Institute of Science \& Technology ("KIST") and the Korean Association of Science \& Technology ("KAST"), developed and maintains a functioning small-scale LED Smart Farm. The problem definition is the result of top-down efforts by local government to respond to the needs of the community members. Diabetes was defined as the most pressing problem after a Community Health Needs Assessment projected diabetes to impact $31.3 \%$ of the population of Manitoba by 2026. OCN and Kelsey's SCC team used their public engagement strategy to explore the root causes of this problem and leveraged knowledge of existing and speculative technologies in order to illustrate how to address the issue.

The three parts of the proposal represent a spectrum of governance structures. Farming is a traditionally regulated industry. The 'app market' is self-regulated through voluntary consensus mechanisms governed by the application developer community. Where this proposal is treading into unexplored territory is in the use of commercial drones and (potentially autonomous) electric vehicles, both of which are lacking existing regulatory structures. Innovations beyond existing regulatory capacity are designed to address the stagnation of existing industry processes and as such constitutes a direct intervention by government into the area's existing food economy. Despite lack of regulatory oversight the public consultation process has demonstrated that community members have demonstrated a willingness to engage in and keep up with this new system.

Volatility, Disruption, \& Response Development: The proposal contains two sides which are connected by the overarching theme of food security and community health. On one side is food production, with the SMART farm capable of operating independently, as it has been doing, to provide the community with fresh produce. On the other side is food logistics, with the proposed ordering and fulfillment technologies reliant upon the farm to feed the community.

Production of fresh fruits and vegetables through new innovations is already a proven concept and does not present much risk. Scaling the farm to meet demand will present a challenge which is accompanied by increased economies of scale to justify the initial expense. If the community becomes too heavily reliant on the farm or on technology mediated access to food, acute shocks could lead to catastrophic failure. A key component of this model is scaling it to other communities to create resilient food networks of Smart Farming communities. 
More concerning are the other two technologies being proposed; autonomous electric vehicles and drones, as well as app-mediated monitoring, education, ordering, and fulfillment. The question that should be asked is why a lower-fidelity solution, such as using existing fleets and grocery stores, would be infeasible or impractical. Existing systems of practice have withstood limited access to fresh ingredients and survived without an app. Mobile applications are expensive to initially develop and deploy and are prone to deprecation whenever devices or operating systems are updated. If an app is to be the residentfacing interface then it needs to be designed to be as resilient as the systems being replaced. Similarly, electric or autonomous vehicles and drones represent a large capital expenditure and infrastructure rollout for currently unproven technologies. The proposal cannot simply procure a fleet but must build capacity to support the vehicles and repair them. Conversion to these systems does contain the possibility of positive environmental and economic impacts by reducing the community's carbon footprint, reducing food delivery costs, and promoting a sustainable food economy within the tri-council area. However, relegation of food culture to an app and the development of supportive electric vehicle, AV, or drone infrastructure has undiscussed social and urban impacts.

There is much at stake when it comes to the impact of systems failures or service delivery interruption. The proposed system is susceptible to all three forms of infrastructure failure; common-cause, cascading, and escalating. Each system component is heavily reliant upon the electrical grid. Cascading systems failures will occur if the SMART farm goes offline. Escalating logistics and waste issues will occur if the app is rendered non-functional or the the autonomous fleet is unable to operate due to weather, maintenance, or software issues.

A scenario in which the community has access to fresh, locally grown produce delivered in a convenient, environmentally friendly manner that accomplishes the goal of reducing diabetes is highly desirable. However, this proposal has relatively shallow leverage in pursuit of intervening in a system by modifying material flows and buffer stocks with Lang (n.d.) citing that much of the work done to address food security focuses on production and distribution solutions which lack the, "power to change the rules of the system". By augmenting these technologies with data-aggregation predicated on monitoring the community's health in pursuit of the systems goal the initial leverage accomplished can shift the existing food paradigm within the community and produce much deeper impact.

Sovereignty and Governance: The proposal is a groundbreaking neighbourhood-class public-private partnership. Despite constituting a government intervention into the local food industry, by socializing part of it, private sector innovators will play a significant role in enabling government to accomplish the project statement. Unmentioned is how government will partner with private industry in planning for Smart Farm expansion, establishing AV infrastructure, or who will ultimately be in charge of fulfillment. How government collaborates with technology vendors on these matters must be addressed to de-risk the project, develop state capacity, and use partnership agreements to ensure successful long-term operation.

The collection of highly sensitive data mentioned in the proposal should also be reconsidered if government's intention is to hand off the project to a private or public-private operator, or if there are interstitial technologies being used to collect, process, or analyze this data. The proposal notes that it "seen as advantageous to leverage data collection at both the individual level and the community level [...] heart rate, blood sugar, and body temperature biometrics can all be 
gathered with the use of minimally invasive wearable technology and gathered in real-time and stored on a community server" (p. 4)

The proposal further speaks to linking this data with food consumption patterns (p. 10). Given the proposal is illustrating a model wherein highly sensitive data is collected and aggregated via third party applications and wearable technology, and given that there is foreign South Korean involvement in this project, how data and access to it is governed is crucial to maintaining the privacy of the community's residents and government control over how this data is used. The inception of this project stems from government access to health data, but that is a very privileged relationship predicated on social contract, law, and political processes (Sieber by way of Wylie, 2018). A next iteration of the proposal should outline how the collection of personally identifiable and community health data is packaged into a civic data trust. To be sure, a data trust is not a final solution but can be the catalyst for a public education and consultation campaign that starts a meaningful conversation regarding what is being collected, who has access, and how it is being used to advance the public interest.

Technical Maintenance: Maintenance strategies for this project are not discussed in the proposal but need to be considered before the project moves forward. The proposed system is complex, with interdependencies requiring consistent maintenance across the system and each technology requiring its own unique form of care. Smart Farm technology, at the core of the project, requires attention to be placed on Mattern's dust based wear and tear (2018) within the farm as, unaddressed, these issues could lead to internal disruption. Complications due to the interiorization of farming will manifest first in organic 'wear and tear', clueing farmers into where the system is not functioning as it should. External disruption of the farm would occur due to electrical grid or other service delivery failure. As such, backup generation, water, and material storage needs to be available on-site as a redundancy measure. Deprecation of this farm is expected. Federal and Korean funding, by way of KIST/KAST, should be considered non-renewable. The farm's operators need to work with local government to ensure that cash flows from food sales are capable of covering the costs to operate and upgrade the farm in order to ensure it continues to meet the community's needs.

The consequences of AV maintenance and winter operation, Mattern's cracks (2014), are discussed in Chapter 5.2.1. However, fleet management is separate from individual vehicle management. Disruption or deprecation can easily occur as the vehicles near the end of their manufacturer's support cycle. Physical maintenance capacity needs to be met with self-driving software maintenance to avoid Mattern's corruption (2018) based wear and tear. Within their warranty, AVs can expect OTA updates, but public sector engineers will be needed to maintain and extend the AVs to continue to suit the needs of the community if no funding is available to replace the fleet.

The app, similarly, requires software maintenance capable of patching it according to Mattern's corruption (2018) and may need to be replaced as mobile platforms change and deprecate older applications. This is the least concerning factor from a systems perspective but a key component to this entire proposal. The app is the only community-facing components of the system. Disruption of the app due to a glitchy deployment or deprecation could lock community members out of the rest of the system. Older methods of ensuring people are still able to access food need to remain in place to ensure this level of redundancy is met. 
Cultural Continuity: Food is not just logistical but cultural. Farming is not just industrial but holistic. One cannot simply sterilize a process of its culture and expect industry to survive. Throughout this proposal little mention is made of existing local or regional agricultural traditions, restaurants, markets, or dishes. The proposal posits an ambitious plan for improving the food security of a community to ensure it stays alive but makes little mention of how the same technologies can be used to advance a food culture that will make the community thrive. As Robidoux, Mason, and Wade (2017) detail, the restoration of land-based cultural food practices found in indigenous populations is vital to developing resilient food ecosystems. The researchers contend that disruptions to food culture and food education, which lead to chronic health issues within northern, is a direct result of colonization. As such, employing communitybased participatory approaches to restoring food culture in tandem with food production and food logistics will further the systems goals of food security and diabetes reduction.

The viability and geography of community members is taken as non-negotiable within the proposal but their food culture becomes technologically mediated. The proposed technology will have a gradual rollout as existing food fulfillment contracts are unwound and new infrastructure and food production facilities are spun up. The sudden availability of healthy, fresh fruits and vegetables needs to be matched with community support to help families adjust to using these ingredients. The app, as part of this infrastructure, the industrialization of SMART farming techniques, and the vehicles that whizz by to deliver food can be thoughtfully used to empower cultural formation. Based on the data presented by the $S C C$ team it is clear that a cultural transformation must occur in unison with the proposed technological shift for produce supply. In the outlined scenario, food education and agency has been delegated to the machine. This if fair for lower-level metrics and day-to-day logistics management, however this delegation needs to be augmented with higher level human initiatives in order for this proposal to modify existing conventions that led to a public health crisis. This could include in-person events that highlight seasonal produce, batch cooking parties, and skill development workshops that engage people to take advantage of the resources being created.

\section{What Happens When The Innovators Move On?}

Direct government intervention into the food economy cannot continue in perpetuity, similar to how it is expected that innovators will move on. Initial investment by government can be matched by planting the seed with local food economy actors to absorb and maintain these new innovations over time. The gradual hand-off frees up the public sector to maintain their own momentum to further address additional factors contributing to local diabetes rates.

Guelph \& Wellington's Smart Farming SCC proposal is also predicated on food-security and emphasizes the need for an internalized circular economy within the urban-rural area. The proposed goal is called $50 \times 50 \times 50$ by 2025 and seeks to increase access to nutritious, affordable food by $50 \%$, create 50 new circulator businesses and collaborations, and increase circular economy revenues by 50\%. To accomplish this Guelph and Wellington propose developing civic infrastructure to create a Smart City food economy lab; in effect placing the onus on the private-sector to deliver on the promises of this technology. The effect being that the proposal sets up the city as a laboratory for a circular food economy but cedes much 
of the technical decision-making and motivation for accomplishing the goal to the private sector.

Unmentioned in the proposal is how the industrialization and interiorization of farming modifies land-use planning for agriculture. Farm designated lands are a heavily protected land-class in most planning regimes. This is a technical aspect and a cultural aspect if one is to consider the changing nature of farming. A farm designation may need to begin considering an FSI or FAR designation that allows for built densities that far exceed the area of the land-mass to encourage developments that create tall vertical farm towers. This may not present a challenge in the tri-council area but will need to be included in a broader resiliency network rollout.

\section{Living Laboratories - City of Vancouver \& Surrey}

Collision free multimodal transit through smart mobility and autonomous vehicles sounds promising, with the municipalities defining their challenge statement as,

"Surrey and Vancouver will implement Canada's first two collision-free multi-modal transportation corridors, leveraging autonomous vehicles and smart technologies to demonstrate the path to safer, healthier and more socially connected communities while reducing emissions, improving transportation efficiency and enhancing livability in the face of rapid growth and traffic congestion. \#SmarterTogether" (Vancouver \& Surrey, 2018, p. 3)

The proposal looks to establish two sensor-rich corridors that enable self-adjusting streets and groundbreaking innovations in transportation technology. Unexplored is how this enables social connectivity and how collision-free transit will be ensured beyond an AV's decision-making ability.

Innovation Policy \& Advocacy: The Vancouver \& Surrey SCC proposal is a combination of the desire to proactively achieve their Vision Zero goal of zero traffic-related fatalities and reactionary speculation based on the promises of autonomous vehicles ("AVs") as a form of public transportation. Given the contained and explicitly experimental nature of the proposal within two corridor study sites, this level of speculation advances the city's goals, rather than hindering them, and is not detrimental to the state of the project. As a municipally orchestrated pilot program the project remains integrated with existing planning processes and the governance structures of the City of Vancouver and City of Surrey's public transit services. The exercise, in partnership with publicly funded institutions and private sector advisors, addresses lack of innovation in this sector with a sweeping plan to comprehensively transform transit. The plan proposes a balanced rollout schedule which should allow stakeholders time to address and absorb new technologies. It is advised that rollout phases be based on absorption and capacity milestones as opposed to a timeline-based schedule, which could lead to rushing engagement and development plans.

Volatility, Disruption, \& Response Development: The proposal encompasses four technical interventions into the transportation corridor; the deployment of AV shuttles, installation of smart mobility infrastructure, platforms for real time and historic data analytics, and an enhanced user experience regime through digital and public realm interventions. Disruption of the power grid would have common-cause impacts on the functioning of the transit corridor as all nodes of the system are reliant on electric power to operate as intended.

However, two end-points of the system, the AV shuttles and app/physical wayfinding do not necessarily rely upon the middle two nodes of the proposal, such that cascading failures (due to shuttle failure) or 
escalating failures (due to app failure) are not likely to occur. Similarly disruption of the Smart Mobility infrastructure, if deployed correctly, will not necessarily disable the shuttles and the app can be programmed to make alternative suggestions in the case of failure in other parts of the system. The discussion in the brief regarding smart mobility infrastructure that can change streetscapes is not dissimilar to the King Street Pilot project in Toronto which opened up more of the street to pedestrians, but at the cost of local businesses making claims about decreased revenues (Fox, 2019). The social and environmental impacts of having safe, revitalized, and high efficiency transit corridors is an excellent outcome for this project. The hope is that long-term data analysis can also focus on the urban and environmental impacts of the project, as both of those are unknowns not addressed in the brief.

The proposed technology elevates the system by introducing new information structures for making the transit corridor safer and more efficient. The proposal is on the cusp of having deep leverage if the system, through analysis, can grow to truly rethink how autonomous public transit functions, similar to how our roadways changed from accommodating horses to being upgraded for cars. Data gathered, expertise from operation, and wisdom from maintenance can be leveraged to advance a paradigm shift in AV transit.

The proposed systems change is being rolled out over three phases; Foundation - the initial installation of the sensors, Integration - the connecting of new and old systems to issue and read multiple data sources in a standard format and, Application - the activation of content and service delivery as well as the ability to change the corridors to meet demand. This incremental approach to deployment allows for growing pains to be addressed as they emerge without compromising other reliant systems. Scenarios that need to be watched closely and mitigated are ones where too much autonomy and rigidity hamper human vibrancy. The transit corridors simply cannot become sterile people tubes that prioritize efficiency above all else. They need to be dynamic, civic, and celebratory in a way that honours and amplifies human culture as much as it improves transportation safety, as outlined in the proposal statement under, "safer, healthier, and more socially connected communities" (p. 3).

Sovereignty and Governance: The proposal is a groundbreaking infrastructure upgrade within very specific transit corridors. The decision to pilot the technology de-risks the deployment and enables the participating transit authorities to build capacity in the technologies before rolling them out to the rest of their networks. The speculative nature of both autonomous transit and transforming streets needs to be incorporated into long-term infrastructure planning in order to bridge from being a novel experiment to being the 'business as usual' option for transit upgrades throughout Vancouver and Surrey. This is no easy feat, as discussed at PoliComm 2019, "In the policy world we are great at start-ups (pilot projects) but not very successful at scaling the good ones \#policomm” (Kearney, 2018). A successful mass-scale deployment will require robust policy prior to beginning this pilot and the development of a highly interoperable system that does not bind the municipalities to the original manufacturer, or any one autonomous vehicle or public realm 'smart' infrastructure vendor. Questions 4 and 6 of the proposal make it clear that interoperability is a key component of the procurement process, however this is undermined by a new Call for Innovation procurement process (Vancouver \& Surrey, 2018, p. 9). It is unclear why established procurement systems are being abandoned for a new 'flexible' process except that privatesector actors did not like the existing process. It is also unclear what exactly has changed. From a sovereignty perspective this is concerning. Procuring new technologies already presents significant risk to 
municipalities, allowing vendors to influence and rewrite the established processes by which such tech is procured is not in keeping with best practices reflective of good governance.

Technical Maintenance: The proposal speaks to the need to integrate with existing infrastructure but does not speak to the increased requirement for maintenance that comes along with higher fidelity infrastructure. The proposal includes physical assets prone to Mattern's (2018) rust, dust, cracks and corruption with Smart Streets infrastructure requiring maintenance not previously required by non-IoT bollards and public realm installations. Similarly, AVs require intensive mechanical labour beyond that required by their predecessors as detailed, 5.2.1. This is the first instance where rust is triggered and it speaks to the systems and infrastructure perspective of this proposal and why it is so important that the proposal is a pilot vis-a-vis a citywide deployment. Dust in the streetscapes, cracks in AVs and Smart objects, as well as corruption in the data and code all add up to a system that is susceptible to rust.

The most physical and cutting edge components of this project are also those most prone to failure via dust and cracks (Mattern, 2018). These being the AV shuttles and the Smart Mobility infrastructure. Autonomous vehicles currently have very little in the way of regulatory frameworks or best practices (as mentioned by the proposal). The proposal identifies the need for a robust infrastructure plan to support the AVs. To make maintenance more effective, charging stations could be able to run an automated digital and physical diagnostic, independent of the vehicle, while it is being charged. Due to the sensitivity of AVs to wear and tear, these stations should work to identify maintenance or mechanical issues as they develop and direct the vehicle towards a larger maintenance facility for manual inspection and recalibration.

The proposal identifies the need for the development of an AV regulatory framework and set of standards. This ratification of AV guidance represents the perfect opportunity to outline interoperable, openspecification maintenance standards for the AV shuttles being procured. Attaching such a system to an open-data system as proposed would allow community members and local start-ups to study the impact of mass scale civic use AVs prior to mass deployment. The hope being that local labour could move beyond reliance on the original innovator and bring AV maintenance into the open-data realm, spawning tertiary markets and businesses, similar to our 'aftermarket' automobile industry today.

Cultural Continuity: The systems intervention proposed is being phased in with data being collected in the first phases helping to inform the physical interventions of later phases. The new features of the system allow for streets to self-adjust and dynamically change based on use and traffic conditions. This is at once a benefit and cause for concern. The ability to broaden pedestrian sections of the streetscape can empower cultural formation by creating new spaces for social experiences. This gives space back to the pedestrian public but the process of creating space needs to be done in a way that enhances the livability of the area and creates moments for social connection. The extent to which the decision to change the streetscape is delegated to the autonomous system or if a consistent schedule of streetscape changes will be established. How resident interaction with the transit corridors is negotiated is a point of concern with few perfect answers. Experiences mediated by the app or the machine must also account for non-users such that the transit corridors cannot become hostile to non-participants or vulnerable populations. Saval's (2019) foray into the world of app-mediated transportation speaks to how public life may actually be erased by evolving forms of transit rather than be empowered by them, 
"Think of the experience of waiting for an Uber driver, in which you follow a single vehicle making turns on an empty Google Map. Everything is evacuated from the picture except for streets: there is nothing standing between you and the vehicle but time and empty space. For a consumer, the image is ethereal. But the streets are actually full of buildings, people, and other cars. [...] The app's interface - that empty map - declares its priorities: the individual, the vehicle, and a place to be. It erases public space and public lives. The public good is not far behind." (Saval, 2019)

This erasure of civic compassion needs to be counteracted by the regulatory and planning processes established around this system. Similarly, 'collision-free' for whom is the prevailing question. A recent death-by-AV in Arizona (Griggs, \& Wakabayashi, 2018 and Bliss, 2018) details the issues that arise when we assume machines to be perfect and humans perfectly obedient. Similarly, AV regulations introduced in the State of Illinois' Autonomous Vehicles Act, modifies how collisions are to be legally considered, "Provides that liability for incidents involving a fully autonomous vehicle shall be determined under existing product liability law or common law negligence principles." Humans cannot be blamed when the informality of life, as it occurs, runs up against absurdly logical and rigid systems. The cultures, vibrancy, and unexpected nature of our cities cannot be compelled to comply with linear, sterile autonomy simply for the sake of our safety.

\section{What Happens When The Innovators Move On?}

Cities as laboratories is a seductive concept and the siren-song of collision-free transit is compelling. However, we cannot assume that the technology itself is capable of delivering upon this guarantee In exchange for being as accommodating as possible municipalities are given first access to cutting-edge technologies. Theoretically this gives them a socio-economic boost by attracting knowledge-sector labour and investment. Failure, however, is part of experimenting, and the majority of Smart City experiments undertaken will be quite novel in nature. Cities that are relying upon adopting the innovations trialled locally as infrastructure need to be aware that innovators are not tied to the city, in fact the opposite may well be true. It may be far simpler for an innovator to unwind itself from an experiment than it is for the city to do the same. The Vancouver and Richmond proposal was selected due to the proposal extending beyond the "city as a laboratory" model, directing experimentation along a specific vector and civic purpose. In this case, the participating municipalities have already made decisions regarding what infrastructure is to be adopted and deployed. The innovators may remain to see the corridor pilots through, but the real challenge for Vancouver and Surrey will be designing a complex policy and planning framework to transform the pilot project into a city-wide system.

\section{Disaster Response - City of Richmond}

Richmond is looking at disaster mitigation technologies that can also serve a dual-use for empowering everyday interactions with government services, defining their challenge statement as,

"Richmond, an island city with a rapidly growing and diverse population and home of nationally significant infrastructure and government services, requires resilient physical and virtual platforms that are integrated seamlessly across all levels of government to enhance quality of life in day-to-day activities and minimize community impacts from major disasters." (Richmond, 2018, p. 7)

At one metre above sea level the City of Richmond will become increasingly exposed to changing weather patterns requiring increased attention to be paid to the minutiae of changes in flood patterns. 
Innovation Policy \& Advocacy: The City of Richmond's $S C C$ entry outlines the real and present danger of flooding and catastrophic events as well as their socio-economic impacts on the community itself, the Vancouver airport, and the broader regional and national economy wherein Richmond acts as a Pacific gateway into the rest of Canada. The proposed technological solution, while maintaining that it does have merit, reads like a laundry list of industry buzzwords and emerging trends. The potential benefits of some technologies is clear. Big data analytics, assistive technology, IoT, and environmental networks all contribute towards emergency response initiatives and disaster mitigation. However, it is difficult to ascertain why terms such as "payment platforms", "enterprise solutions", or "networks" are being included within this proposal (Richmond, p. 35). The outline discusses leveraging machine learning to help guide decision-making but that is very different from implementation of an artificial intelligence platform which owns the decision-making.

Integration of the platform into existing planning processes is evident throughout the proposal and explicitly discussed with how new learnings generated by the platform can help to inform planning decisions with regard to protecting the vulnerable island city. Upon deployment, this technology's application is very focused on improving the day-to-day quality of life for residents as well as disaster mitigation. How this technology will change long-term planning and development processes themselves as a new form of technology-mediated decision-making will require further consideration.

The regulatory structure of this proposal is unique in that it seeks to centralize decision-making for a disparate array of decentralized agencies, municipal services, and private corporations, as illustrated in Appendix 7. This is compelling in that, if successful, it can increase emergency response efficiency and avoid redundant responses, instead serving a broader service area and resident population. It remains to be seen if private sector participants can be convinced to participate and how formerly self-regulated response plans will adapt to such a governance system. It should be noted that private sector actors would rely heavily on government if the magnitude of a catastrophe surpassed their level of preparedness or response capacity.

This technology is primarily being advocated for by government, but there is a clear private sector influence discernable from the direction that the project is taking, some of the solutions being suggested, and larger corporations being explicitly listed as members of the project's advisory team. These include Radical I/O (a current platform vendor), Telus, TIBCO, and Amaresco. This is clearly a desired change to civic service delivery that is being advocated for by both the private and public sector stakeholders. Given the capacity of all of the listed advisors and stakeholders adapting to the pace of change is

Volatility, Disruption \& Response Development: This proposal is predicated upon reacting to service delivery disruption and systems failure. Based on this project priority solutions to address common-cause failures, such as electrical grid disruption, are most likely baked into the hardware deployment itself. Cascading failures are more of a concern given the centralized and digital nature of decision-making in the proposed system. In emergencies it is impossible to account for all potential possibilities. Unexpected events or conditions that are not included in the system's logic model can lead to disaster responses being performed in error or conducted inefficiently. Similarly, escalating failures can occur if reporting hardware is damaged or otherwise disconnected from the response management system. 
Collectively, volatility from both system disruption and success hinges on the extent to which the agency of the platform supplants human decision-making. The environmental and economic impacts of the project are clear, but the social and urban impacts of such a system are longer-term and more nebulous. How resources are directed and deployed by an algorithm, whether in day-to-day or emergency contexts, has significant ramifications for social and urban aspects of the City of Richmond. The informalities of urban life and human social conditions are impossible to account for in an algorithm to the extant that a decision made exclusively based on sensor data may lead to inequitable, unconstitutional, or tragic results.

The proposed technology reinforced feedback loops and intervenes within the system at a shallow leverage point. The increasing volatility of our weather systems will require solutions at an increasingly higher leverage point. It is advanced that the City of Richmond project lays the groundwork for future projects to be developed upon [come back to this; dispensable areas and communities]

Two scenarios that emerge from this system need to be planned for. One where the machine is making choices based exclusively on data collected from the platform and one where the machines is informing a human team of decision-makers allowing for more efficient response development and resource deployment.

Sovereignty and Governance: Richmond's proposal brings together disparate actors in a new form of the Public Private Partnership model ("P3"). The project life cycle involves a build and deliver partnership at its inception but given the operational systems goal is to coordinate emergency response efforts from both public and private sector actors, this model will also require long-term operating agreements between many of these groups. The P3 process under consideration elevates a New Public Management horizontal model ("NPM") to that of an exercise in state capacity building that places the state as the central authority in this system, via an autonomous decision-maker, reminiscent of Clarke's (2018) discussion of this concept. The project scale is citywide but the risk to private industry vendors is minimized based on the digital-service delivery nature of the project. Data governance and platform ownership will be a key aspect of any partnerships or vendor relationships in this project. The City of Richmond's quality of life vis-a-vis emergency response matrix shows the potential dual uses of this platform. Similar thought must be put towards how other actors, plugged into the system, could be using the data and relationships generated by this system.

The deployment of this system and the collaborative opportunities of working with multiple groups of stakeholders should be treated as both an opportunity to cement the state as the central authority in emergency response development and platform analytics but also be treated as a chance to revisit planning process for civic technology rollouts and flood mitigation. As operators of this network the City of Richmond needs to feel comfortable operating the platform as it was delivered but also build upon and improve it as new methods of operation and analysis are required.

Technical Maintenance: The spectrum of wear and tear to our understanding of deprecation changes with a proposal such as Richmond's, wherein the system is designed to defend against disruption. The crux of the initiative is a maintenance and resilience oriented platform. This raises the question of to what 
extent can technology qualify as a maintainer. The system is being designed to monitor different aspects of civic life and flooding behaviours, as such the collection and analysis of data must manifest in physical maintenance practice. Mattern's (2018) modelling of maintenance brings forward both implications of required dust and corruption in this proposal with the physical deployments of sensors and infrastructure needing to be integrated into existing maintenance schedules and the learning algorithms requiring constant work to address the appropriate interpretation of data.

There is a general divide between the execution of physical and digital labour in this project. On the ground maintenance workers will need to be trained in sensor technology in order to mitigate systems disruption and address systems failure. To ensure continuity, the City of Richmond should not have to rely on the technology vendors to perform a service call during or after an inclement weather or flooding event. Prioritizing repairs to the system and physical changes to the natural or built topographies of the city should be considered one and the same. This could include how resources are directed to clear blocked roadways, inspect resiliency measures, and open the parks and paths after flooding. Engineers maintaining the digital side of the system will need to address how the body of data being used for response development grows over time, and how the influences outcomes.

How the city and the system are maintained, and which receives more attention, triggers a power dynamic of how waste is defined and how civic priorities are determined. As will be expanded upon in the following section, the extent to which the machine participates in decision-making regarding its own maintenance and repair during acute shocks and chronic stressors can have city-wide ramifications on who benefits from its deployment and who does not.

Cultural Continuity: The geographies of human settlement in the City of Richmond is considered nonnegotiable by the proposal and may come to be a fault with the project. Richmond's one metre elevation above sea level leaves it in a precarious position as weather patterns become more extreme. This project raises serious concerns regarding the extent to which we consider the location of built-up urban human settlements to be non-negotiable. At what point do the maintenance requirements of such settlements become unviable enough that planners need to consider unwinding parts of cities rather than further reinforce and entrench them with increasingly expensive resilience measures?

The combined quality-of-life and emergency operation based dual use of the technology should give residents the opportunity to integrate the myRichmond platform into their lives prior to use during high pressure situations. The City of Richmond's desire to make the resident facing platform bridge language barriers and use mobility systems to enhance daily life should be applauded as an easy way to embed these systems into the daily habits of Richmond's residents. The concern with such a system is how much decision-making is being delegate to the machine's ability to analyze data rather than to local culture's ability to prioritize and set guidance. From Smart Streets to realtime machine learning based traffic management, AI based chatbots to community asset demand prediction the City of Richmond has thoroughly embedded algorithm based decision-making throughout their proposal. The extent to which such decision-making can empower human initiative rather than supercede it must be constantly (re)emphasized to those working in context with the system. O'Neil's (2016) demonology of how such algorithms foster inequality and threaten democracy can simply not be allowed to play out. Humans may need to intervene with emergency decisions that, to a machine, appear to be illogical but equitable, 
inefficient but brave. The machine's ability to prioritize and de-emphasize must always be subjected to intense human scrutiny.

\section{What Happens When Innovators Move On?}

The City of Richmond needs to be able to protect itself beyond the 'new normal'. Our climate will continue to change and, if successful, this deployment will need to be able to change and evolve with it. Today's sensor grids may be tomorrow's water line. As identified earlier, Richmond needs to ensure three components of this plan are secured to ensure its continuity as a city and as a community.

The biggest concern with this project is its impact on cultural and social continuity. The system is purpose-built to protect itself, preserve infrastructure, and deploy emergency response resources. It is not, however, designed to help individuals beyond any preparedness/response guidance that the MyRichmond app may give to residents. No apps, infrastructure projects, and algorithms can replace social cohesions and community trust, especially if the power goes out. Extreme weather and infrastructure events highlight the best in people and the worst in our municipal systems. Toronto's 2013 Ice Storm (Agro, Apollonio, \& Cheung, 2013) and 2003 blackout (McNalty, 2019) both stand out as examples of where disaster responses based on human initiative and community cohesion far surpassed system resilience. Reliance on the system proposed for the $S C C$ cannot continue past Richmond's ability to maintain it. Funding for the system cannot stand in lieu of continued funding for the maintainers. Increasing the technical complexity of a flood mitigation and emergency response system must also be matched with increased financial support for existing assets.

\section{In Summary}

A school, a farm, a bus, an emergency response; each proposal is intervening in one of the basic elements of modern human settlements with the objective of effecting long-lasting change. Viewed as objects, or even discrete networks, the technical exercise of upgrading them seems simple enough. Put one online, make one indoors, let one drive itself, let one tell us how to respond to a flood. However, these objects do not exist in vacuums, but in complex systems; our education system, our food industry, our transit system, our ecosystem. All of these systems layer on top of one another to empower, feed, move, and protect human life and our cities. If one system is not maintained, they will all be impacted. "Complexity is descriptive of systems, especially dynamic systems, where the elements of the system interact with each other in subtle ways. Those interactions may bring different results in the short term versus the long term, because complex systems evolve and adapt. Sometimes that evolution is surprising" (PwC \& ULI, 2018, p. 3). A key tenet of the Smart Cities Challenge is that the systems being funded be scalable to other municipalities. Examination of the localized impacts of new technologies and their material impact on new cities, if we can maintain them, begins to point to what the consequences of their adoption would mean.

In the case of Biigtigong Nishnaabeg (Pic River First Nation) the ability to go to school online, in any community, will lead to the need to re-evaluate how our current and future educational infrastructure serves students, knowledge dissemination, and social cohesion. In the case of Town of the Pas, Opaskwayak Cree Nation, and the municipality of Kelsey the ability to urbanize farming at a mass scale close to market will change how we plan for farming outside of cities and designate employment lands within them. In the case of the cities of Vancouver and Surrey mass-scale adoption can bring pedestrians 
back to the centre of transit corridor planning, rather than pushing them to the periphery through autonomous transit and Smart Streets infrastructure. In the case of the City of Richmond we are forced to reckon with the long-term viability of human settlements when changing weather patterns begins to point to the need to displace communities in order to save them.

Each of the cases faces continuity and maintenance challenges that are unique to both their community and the proposed Smart City intervention. Between all of these cases are commonalities which can be addressed through process innovation, prolongation considerations, and proper planning practices. 


\section{Ch 5 Conclusions}

The five lenses each explore a different topic of focus for each community's relationship with the proposed Smart City intervention. Discussions regarding the legibility of a solution, the readiness of a community, government's ability to support the system, the maintainer labour required to sustain it, and in doing all of this, the community's ability to be empowered by such an upgrade.

To link back to Deacon's (2018) Google Fibe example, KCK and KCM advocated for the deployment of Google's Fibe technology. Its benefits were legible. The cities leveraged resources to ready the community for the technology's arrival. Government established a council to guide and deploy the technology and in so doing developed state capacity in the sector. Evidence of the community's empowerment was so powerful that it lead to other communities visiting the region to learn more about how Google Fibe could be scaled to their city in pursuit of developing their own advocacy efforts.

One such community was that of Louisville, Kentucky. The City of Louisville accommodated Google Fiber's initial rollout and experimentation with a new fixed line deployment method. Louisville residents advocated on behalf of the company to their local municipal council in order to secure permission for the install. On the 7th of February, 2019 an acute shock hit the citizens of Louisville, Kentucky; Google Fiber would be unwinding their experiment (Welch, 2019). This shutdown, it was announced, would turn off existing customers' internet access within two months, abandon miles of fibre-optic hardline, leave the roads in a state of disrepair and the city in shock. The announcement to shutter the service was announced via a tweet and blog post. As this paper was being written the consequences of what is being discussed were playing out in real time. Residents took to Twitter to ask, in many forms, 'what happens now?'.

The combined academic and media coverage of Google Fibe spans three cities, multiple years, and the entire lifecycle of what is being discussed in this body of research. It was sheer dumb luck that this timely series of events occurred at the time of writing and are reflective of the analysis being performed. Where this Fibe case study faltered was in using past successes to buy room to experiment. Experimentation in a new city failed due to a number of factors, such as the decision to move beyond best practice into unexplored territory in order to cut costs. This points to the need to ensure that scaling a technology revisits what exactly is being scaled in order to ensure that site-specificity is baked into the process. In this case it appears as though it was the Fibe brand and not the successful Fibe service delivery method that was brought to Louisville..

The discrete and site-specific issues identified within the analysis of each case in Chapter 4 cumulatively identify broader overriding elements regarding how cities take over from innovators that are shared by all cases examined. Almost all of the cases fared well in the first lens regarding legibility, as these were municipally initiated projects, but there are outstanding considerations shared amongst all of the cases under the lenses of critique.

\section{Volatility, Disruption, and Response Development - Process + Readiness}

\subsection{Asking Better Questions to Motivate Better Answers}

The Smart Cities Challenge asked all entrants to answer Question 8, "Please describe your community's readiness and ability to implement your proposal successfully" tasking each community with outlining their experience implementing complex projects. Responses spoke to the structures, processes, and 
practices in place for managing and implementing complex projects as well as the municipality's organizational strengths and weaknesses. This question would be a good opportunity for a municipality to holistically discuss their capacity and preparedness for deploying the proposed technology into their community by identifying bureaucratic, technical, and culture barriers to adoption.

Respondents used this question as an opportunity to highlight their government's prowess at tackling large projects. Such a track record should be highlighted as part of the SCC process, but it is only one part of the community which needed to be evaluated in preparation for submission in order to answer the question. Question 8 needs to outline the parts of the community required to demonstrate capacity for the specific project alongside government. In many cases this will need to include innovation partners, private sector actors, domain-specific labour force capacity, and resident readiness, as well as infrastructure restraints.

Demonstration of public consultation requirements, and execution thereof, is included under Question 5 in the RFP. Municipalities used these meetings for problem identification and ideation, however there is little evidence in many of the proposals analyzed that final proposals were run back against the community for endorsement, early adoption participants, or to address resident concerns about deployment or long-term systems adoption. To achieve meaningful long-term support from the community, efforts needed to be made to educate participants regarding the implications and repercussions of systems intervention and disruption. Communities need to be as prepared for volatility as they are for access to new technologies.

The interconnected and interdependent nature of our communities requires that, for systems change to be successful, buy-in from existing groups of actors needs to occur. Disruption and volatility are not sector or vendor specific but, as per Dushenko (2012), have unexpected secondary and tertiary social, economic, and governance impacts. Question 8 could be expanded to encourage later phase consultation processes to promote engagement that focused on community readiness and preparedness. Going through this process is also a good opportunity to explore exactly what impacts could look like. Engagement presents an opportunity for unexpected leaders, those not listed in the documents, to identify where they see benefits or downsides. The recognition of unexpected advocates relieves pressure from solely relegating this forecasting exercise to the framers of the RFP response who are focused on a much broader set of conditions that must be met to successfully apply for and receive funding.

The readiness of a community must also detail the readiness of its dependent infrastructure systems. Some of this can be mitigated by smart systems intervention, as per Clarke's (2018) model, but it is critical that its current state and planned future upgrades be included in the proposal. The imposition of new, heavy systems on existing fragile infrastructure simply cannot lead to long-term sustainability and systems continuity.

\subsection{Addressing Underlying Infrastructure \& Technical Debt}

Upgrades to our existing civic infrastructure systems should be considered a prerequisite for the construction of Smart Cities Challenge projects. The current state of our supportive infrastructure is, at best, fragile. The two most relied upon infrastructures in $S C C$ proposals, our electrical grid and our internet systems, require significant continuous maintenance to ensure constant service delivery. This is a 
perpetual concern when analyzing $S C C$ entries and a persistent source of common-cause failures for the systems being proposed. The technology industry's terms of technical debt and technical inflation demonstrate how these problems of are singular importance when programmers are engineering code, but also serve as important concepts to consider as new systems are built on top of aging ones.

In most of Canada the electrical grid is either operated within the public sector, are public-sector adjacent, or under extreme oversight by government. The cost of electrical service delivery has become a political flashpoint at both the provincial and federal levels of government. Residents simply cannot afford the increased costs of electricity. This impacts our ability to maintain and upgrade the grid to satisfy our appetite for powered devices. Both the fragility and cost of power generation and delivery pose an important problem when considering smarter infrastructures. How are we supposed to adopt and integrate higher fidelity civic technologies, which require significantly more devices to be plugged into the grid, if the infrastructure upon which all of our new systems are reliant cannot be upgraded?

The state of Canada's internet infrastructure faces the opposite problem. Both our fixed-line and mobiledata systems are controlled by a select group of Internet Service Providers ("ISPs"). Net neutrality regulations currently stop these companies from privileging certain types and forms of data over others but the private-sector nature of these organizations means that infrastructure construction and upgrades will be spread unequally between urban and rural communities. Rural communities may be solely dependent upon the services of one ISP and due to this monopoly may find themselves paying higher fees for lower-tier services when compared with urban internet costs and quality. For example, the community members of Pic River First Nation are paying \$50 a month for 5 GB of bandwidth at a peak speed of 5 mbit (Pic River Development Corporation, 2019) whereas the author of this research, living in Toronto, pays $\$ 50$ a month for unlimited bandwidth at a peak speed of $15 \mathrm{mbit}$ (Start.ca, 2019). It should be noted that both of the listed ISPs are third-party Bell/Rogers resellers. The discrepancy between infrastructure development and service costs presents a significant barrier of access and is a source of urban-rural tension regarding the civic prosperity promised by many new Smart City infrastructures. Gallagher's (2017) treatise on the nation-building role of the post-office in America speaks to the importance of equitable access to information infrastructure and its role in building and maintaining a democracy's central nervous system. This serves as an important lesson for how we conduct plan new infrastructure systems today.

The Government of British Columbia has begun to invest in rural access to high-speed internet, with Grewal (2019) documenting the mid-March announcement of a $\$ 50$ million investment to build out highspeed internet infrastructure to 200 rural and indigenous communities within the province. The CEO of the Northern Development Initiative Trust tasked with the deployment stated that, "better access to highspeed internet helps close the digital divide between urban and remote areas" with such access giving communities the ability to grow their communities, attract new residents, and provide the infrastructure necessary to hinder out-migration. Similarly, the Federal Liberal government has recognized the need to invest in internet infrastructure across the country, with the 2019 budget dedicating $\$ 1.7$ billion over 13 years in pursuit of giving all Canadians access to high-speed internet by 2030 (Cochrane, 2019).

Lacking domain expertise in each field, it is difficult to comprehensively speak to the state of each critical infrastructure piece, but this is not a concern unique to this paper. As identified by Gendron \& Rudner 
(2012) both of these systems constitute critical national infrastructure services that must be upgraded and maintained. It would be gauche to suggest that our internet become socialized. However, it is critical to address the importance of equal access to, and ongoing investment in, infrastructure when engaging in city-building exercises locally and nation-building exercises federally.

\section{Technical Maintenance + Maintainer Labour}

Planners and maintainers need to be aware that no object comes without its world, and no world comes without its maintenance (Denis \& Pontille, 2017). The transformative potential of autonomous vehicles is mentioned repeatedly between the OCN-Kelsey and Vancouver-Surrey proposals; the social and environmental benefits of electric-autonomous vehicles is self-evident. The Richmond proposal is similarly reliant upon sensor-based technologies to inform an AI decision-maker and the Biigtigong Nishnaabeg proposal has very discrete maintenance requirements. All of these systems are new worlds with new maintenance standards, however vehicles and their required maintenance is both the most studied infrastructure in this field and the most comfortable metaphor for many readers.

The wintery anecdote of former Ontario Finance Minister Greg Sorbara (2019), illustrates how a hostile environment combined with operator error and lack of infrastructure can lead to near catastrophe. This personal story of frustration sets the stage for a growing body of discourse regarding how complicated maintenance on intelligent systems can be and how much specialized labour is required. To carry on the Tesla commentary, but not to specifically call them out, Higgins (2019) explores how a lack of regional infrastructure and lack of 'after-market' parts (due to the proprietary platform) can make local repairs difficult and lengthy, inhibiting both vehicles and technicians. Reliance on first-party support from a company headquartered on the other side of the continent means that repairs of for Tesla vehicles can take far longer when compared with other automobile manufacturers.

Sensor-rich vehicles that provide 'semi-autonomous' driver assists, vehicles which are incrementally marching towards full autonomy, are highly sensitive. Quain's (2019) explored the substantial impact that smarter vehicles have on required labour for repairs. The use of radar, camera, ultrasonic, and lidar technologies to accomplish semi-autonomy means that any slight adjustment to a vehicle, either through damage or maintenance, can require a complete re-calibration of the vehicle,

"Cameras nestled behind the windshield, radar sensors hidden in the front grille and ultrasonic components embedded in bumpers work within narrow tolerances. A seemingly minor impact can knock them out of alignment. And as vehicles — and drivers - rely on these systems more and more, tuning these components properly can be a life-or-death matter" (Quain, 2019).

This is a far cry from how maintenance on vehicles started out. Successive generations of vehicles have restricted the owner or operator's ability to repair vehicles themselves, requiring the services of authorized technicians to delve into specialized (but still standardized) error code messages through the OBD2 terminal. Maintenance and repair practices have now been significantly changed, lengthened, and made more complex by semi-autonomous assists. The additional step by step process of how new repair processes are performed is begins with what was previously a half-hour fix,

"Fixing a cracked windshield used to be the kind of repair a technician could perform in a few minutes in your office parking lot while you were at work. Today, the same repair could take hours and require expensive computerized equipment in a special facility [...] The 
vehicle also ha[s] to be driven at over 50 m.p.h. for at least 20 minutes — in clear weather enough time for the sensing system to confirm that it was correctly calibrated." (Quain, 2019) For any repair shop, the ability perform this level of maintenance presents a significant investment into educating mechanics on new processes and renovating the shop to meet the manufacturer specification. Owners, similarly, are covering the cost of higher repair bills due to the amount of labour required, if the repairs can even be performed locally.

Vehicles are the most convenient metaphor for exploring the topic of how the performance of labour changes with smarter technologies, but points to the broader maintenance worlds that these systems bring with them, especially if the technology is being procured and maintained by a municipality. Whether selfadjusting street systems, sensory systems, or autonomous fleets, how the tertiary requirements of these technologies is accounted for in the RFP must be outlined both financially and with regard given to the availability, education, and required training of local talent.

\section{Systems Sovereignty, Governance, and (Self) Regulation}

Vancouver and Surrey's proposal identifies the need for the development of an AV regulatory framework and set of standards. It also emphasizes the need for procured systems to be interoperable. This ratification of AV guidance represents the perfect opportunity to outline interoperable, open-specification maintenance standards for the AV shuttles being procured. This is significant, as it shows public sector recognizing these 'smart' objects in much broader, interconnected systems, something regulations are still struggling with regarding individual automobiles vis-a-vis 'traffic'. A systems-perspective on $S C C$ proposals needs to operate from a position of access, understanding, and the ability to intervene. What is emphasized in the proposals selected are digital-to-physical interventions that require interoperability between distinct pieces and the ability to be maintained and repaired in order to accomplish and sustain the systems goal. The jump to physical products is where many innovation actors struggle, where existing systems of practice are most experienced, and where the inspiration for this body of research was found. Physical products pushed out into the world by innovation actors tend not to reflect the same level of openness or duty to maintain as the digital counterpart it was designed to work with. Physical products are too easily deprecated and are replaced rather than repaired. This mindset, and distinction in duty of care, between digital and physical, will simply not work for SCC projects.

To borrow a metaphor, the large-scale infrastructures being proposed need to function like the plumbing system in your house. Different labour groups, engineers, designers, and product manufacturers all converge on this tiny, but complicated, series of tubes, taps, toilets, and temperature devices in order to bring a municipally delivered service from the street to your sink. This system is comprised of a combination of agreed upon standards, achieved through industry associations, and system components procured under product specification documentation.

Where many of the SCC proposals differ from established norms, and where there is significant concern, is when entire systems are being proposed and deployed based on sole-source specifications. Many of these speculative technologies are only available from a limited number of companies each selling proprietary solutions. Due to the 'winner-take-all' approach to these products little industry consensus can be achieved. New systems being procured cannot be reliant upon one vendor, with Heinsdorf (2014) citing that public sector contracts generally require specifications to have at least three product options to 
ensure fair and competitive bidding. Sole-sourcing or closed proprietary specifications are justifiable when significant engineering work is required to ensure compatibility with an existing system. (Ibid.) The shift away from both standard procurement processes and performance or prescriptive based specification processes (Goren, 2016) towards sole-sourced closed-specification models is a marked change in direction away from processes that ensure repairability. Achieving voluntary consensus on standards from industry is a key component to sustaining the improvements achieved through new technologies,

"Standards create the foundations for a better world [...] In an age of breathless enthusiasm for the new and 'disruptive,' it's worth remembering the mundane agreements embodied in the things around us. It's very ordinariness and settledness of standards that enable us to survive, and to move ahead" (Russell, 2019).

New innovations and their creators rarely subscribe to, and thus struggle to be governed by, established processes. When one can universally activate a service in any community, little regard is given to the required site-specificity of any one locale or industry. The $S C C$ entries are municipally initiated, civically transformative, and modify existing systems that do fall within the realm of planning and our established best practices of procurement. These case studies are an opportunity for the long-term-oriented strengths of the planning and construction professions to make a case for our inclusion in the proposal formulation processes.

\section{Advocacy, Legibility, \& Continuity - Considerations for Planners}

Standards and systems set the stage but planners are given agency to pick the site and direct the play for the communities they serve. The systems stability accomplished via labour training, standards, and object specifications must be adapted to specific needs, desires, and ambitions of the context into which a technology is being deployed. A Robertson screw (Canada, 2005) can be put into any piece of wood in any direction, but where, how, and why it is used is an important consideration when assembling a structure. The complexity of this decision-making process and its impact on the built, natural, and social context of cities is compounded when inserting new systems into sites with interrelated and interdependent infrastructure systems. Planners need to entrench themselves within these systems to ensure the short and long-term viability of our communities. The Planner's Responsibility to the Public Interest, 1.0 under the OPPI's Planner's Professional Code of Practice, tasks planners with the need to: "1.3 acknowledge the inter-related nature of planning decisions and their consequences for individuals, the natural and built environment, and the broader public interest;" (p. 14). Such an acknowledgement is further given guidance by the Canadian Institute of Planners (CIP) Statement of Values, specifically:

\subsection{To respect and integrate the needs of future generations}

Every generation builds a foundation for the next one. Planners cannot foresee what the unique needs of future generations will be but we can ensure that they will be able to derive value from the investments that we have made in infrastructure to better our and their quality of life. This is accomplished by planners through ensuring repairability by local labour, inscribing the right to repair/replace/service into infrastructure agreements, and ensuring that procured systems are open enough to be upgraded and extended beyond the life of their original creators. All of this is dependent upon securing funding to service infrastructure over its lifetime such that it is not allowed it to fall into disrepair. The practices of deferring infrastructure maintenance to balance municipal budgets cannot continue to be the status quo. Either new systems are procured with funded long-term maintenance plans or they are not implemented. 
It is also important to ensure that current and future generations have the right not to participate in systems that they deem to be oppressive, exploitative or harmful. The systems that we are procuring cannot assume consent from birth, similar to how we are able to opt-out of certain civic systems without losing access to said systems, We need to ensconce the rights of future generations into RFPs such that they can opt-out of the mass data collection exercises inscribed in many of the SCC challenges. As stated earlier, Data Trusts are a good first step, but are not the final solution for data ethics and data collection.

\subsection{To overcome or compensate for jurisdictional limitations}

The Biigtiigong Nishnaabeg proposal demonstrates that our communities are no longer constrained to bright-line geographic boundaries, they can become ephemeral or digital. The OCN-Kelsey proposal demonstrates that the health of our communities is not confined to physical annual check-ins with health practitioners but can be improved and maintained through a complex system of industrialized and cultural processes which can be digitally mediated, monitored, and used to inform future planning efforts. The City of Vancouver and The Town of Greater Sudbury demonstrate the extent to which data may drive the reshaping of the public realm and civic domains. The City of Richmond and Simcoe County demonstrating the extent to which we may need to rely upon digital architectures to inform disaster mitigation efforts and long-term planning to avoid catastrophe. So too are internet-enabled technologies not bounded by any one domain, jurisdiction, or governing body. As much as planners have previously had a role in each of these systems, technology vendors have come to play a similar role by layering a digital topography on top of the existing human, built, and natural geographies of our communities (Lister, 2012). Planners must be tasked with considering statute 1.3 as also pertaining to the interrelated nature of emerging infrastructure systems and technocratic decision-making. These issues impact individuals, the natural and built environment, communities and cultures, as well as the broader public interest. The universality of these technologies and the radius of their impacts requires that planners be willing to look outside of their specialization or jurisdiction. This, at the surface, appears as a heavy burden to bear but if planners refuse to own our role amidst these systems others will and they are not bound by a professional responsibility to serve the public interest. Planners can accomplish this by researching how new technologies being deployed in other communities could be applied to their own and monitor what steps were taken to mitigate adverse impacts.

\subsection{To value the natural and cultural environment; to articulate and communicate values; to recognize and react positively to uncertainty}

Technology can provide a catalyst for massive short-term gains towards solving a defined problem but it will also, on a longer term basis, transform the fabric of the communities in which the solution is being deployed. In essence, the proposed technology is supposed to create, change, or benefit, a Smart City. Given the civic development context of this work, an outstanding question is 'where are the planners?'.

Writing in the Ontario Planning Journal, Horne (2018) comments on the great openness required by communities in order to apply Smart City thinking without sparking instability, stating that the, "role of the professional planner is critical to supporting community vitality as technology evolves" (p.1). Horne argues that a lack of of understanding is furthering the slow decline or instability experienced by communities when it comes to adopting new technologies. 
This lack of understanding does not just stem from a lack of technical knowledge but from the lack of consistent thinking about how technologies will be applied and what they will accomplish. Long-term thinking regarding technological transformation can be baked into existing systems of practice that guide future development. Official Plans do not just designate land use. They illustrate the future of a city and its precincts over the long term. The document is intended to paint a narrative that guides development, promotes specific initiatives, directs investment, and gives voice to a community's values and goals by outlining specific principles. Every Official Plan considers the subject municipality from environmental, physical, cultural, and social contexts. How new technology is adopted must be paired with the social consequences of adopting such a technology. How the technology meshes with the narratives. How technology extends or interferes with this vision must be reconciled, either through policy or direct intervention. Yet at the same time, the pace of change being witnessed is "moving at a speed that is good for downloading movies, but less good for planning what we want our future to look like, and who we want it to serve." (Renzetti, 2019). These documents are flexible frameworks, not prescriptive instructions, that recognize old problems in search of solutions. Our ability to set up our plans to empower technological transformation can make all the difference in the world for how we react to it when it arrives and encourages broader civic capacity to maintain it once it is adopted.

Much ado has been made in this research about a municipality's unique vulnerabilities and how its cultural narrative can empower techno-determinism to create a compelling case for the adoption of new technology. The question of how this narrative is formulated in the complex, multicultural environs of some of our nation's largest cities is answered through Official Plans. Documents that are purpose-built to look to the horizon need to consider what, technologically, may be on the other side of it and begin to plan for its proliferation in a manner that benefits us. Referenced earlier was the requirement for planners to not just weather the storm, but dance in the rain. How future uncertainty is accounted for in our current plans can lay the groundwork for ongoing systems stability. Using Official Plans and other visioning tools to preempt disruption can detail the choreography required to turn disruption into graceful transformations.

\subsection{The inter-related nature of planning decisions}

What arose from applying a hard planning lens to these themes in the previous section, as opposed to the soft planning lens in the rest of the paper, is that planners have an opportunity to own this narrative with our existing toolkits. There is a recursive loop of renewal present within our visioning documents that connects cultural continuity back to policy and advocacy. In the development lifecycle of any one product or intervention, the linearity of this process would normally forces us to consider the elements of each technology in an order similar to the one used in Chapter 3. However, in the broader context of cities and communities, the realms in which planners play, "systems happen all at once" (Meadows, 2008, p. 5). The recursive loop of systems change and urban renewal is one which planners have built upon incrementally over generations. The technology is new, but this is not terra incognita unless we allow it to be. The same processes must be followed, the same labour must be performed, and the same duty of care applies.

\section{Awareness To Action}

While speculative in the case studies surveyed, how cities adapt to innovators moving on is a real and present concern for municipalities and needs to become a priority for planners operating within these communities. What emerged was a lengthy conversation but building forward from today, what does this 
look like? How do planners concisely apply everything that has been discussed? What should future research entail?

\subsection{Follow the proposals}

All of the proposals are unique narratives of a community envisioning its future, even if they are not funded. All 130 proposals reflect the collective visions and wisdom of a diverse array of communities across Canada. In that, there is significant value.

At the city scale the cases that end up being awarded funding should be studied as they shift from ideation to deployment to handover from innovators to maintainers. So too, should the proposals that are not awarded funding be studied. What does their response development looks like? How does the municipality pivot, move forward, or abandon the idea? There is significant work that can be done to study how communities deal with the excitement generated by this challenge and their proposals in order to better inform future challenges, RFP processes, and the design of Smart City development projects..

\subsection{Follow through with scaling}

How do plans for scaling the technologies, as illustrated in the proposals, adapt the technology to new locales and what cost-savings measures might be considered? Deacon's (2017) research into the highprofile pilots for Google Fibe in KCK/KCM is contrasted against Welch's (2019) follow up detailing the disastrous results of scaling the infrastructure to the new site of Louisville, KY. If there is meaningful follow through regarding scaling these systems, attention needs to be paid to ensuring that the same level of quality baked into a successful, high-profile pilot is extended to other communities wanting to participate.

\subsection{Deploy investments at scale}

At a national scale it is worth compiling documentation regarding the $S C C$ submissions. How the themes of legibility, advocacy, readiness, governance, maintenance, and continuity are addressed should be analyzed for commonalities across geographic regions and different community sizes. This can point to where there are systemic issues or chronic needs that can be addressed by making investments in developing Smart City systems at scale. Likewise, where there are readiness issues, as identified in section 1.2 of this chapter or other as of yet unidentified infrastructure systems requiring repair or upgrading can direct federal involvement, as part the 2019 budget directive on internet accessibility (Cochrane, 2019).

\subsection{Follow up with communities}

Disappointment is a natural counterpart to excitement. Planners deal with disappointed residents, community members, and advocates constantly as part of our work. It is an undiscussed but pertinent human component adjacent to, and prevalent within, the desire for systems maintenance and continuity. The efforts put into these proposals, the visions that communities were sold on, and the hopes could leave them susceptible to private sector influence if the investments made in visioning do not pan out and convert into actions. On the other hand, continuing with the proposal on the municipality's terms through P3 mechanisms can empower communities to own the narrative of their transformation. Between the two there is a balance. Again there is a stark difference between how communities which achieved SCC funding and those that did not are supported. Communities which are not funded may expedite the P3 
decision-making process in order to avoid disappointing residents or deferring investment in renewal. This decision-making process may lack the patient and methodical analysis required to ensure that such agreements are in the best interest of the public. The visions laid out in the proposals that are funded may not be able to fully manifest the future residents imagined. There is a role for planners to engage with communities in both of these scenarios. Managing expectations is vital to ensuring that disappointment does not overtake excitement which could compromise the realistic deployment and long-term viability of the proposed systems.

\subsection{Prepare communities for innovators moving on}

We do not have the ability to "move fast and break things", truly we never did (Taneja, 2019). Cities by their very nature are immobile, slow moving vehicles. Past innovations have advanced the visioning Smart City technology furthers future innovation. Innovators move on, we need to move past this being a disruptive event, and rather address how we can take over from the project's inception. Planners have the privilege of growing with a community over a much larger period of time. We record shifts in priorities, changes in our cities, and study the consequences of past and current disruptive events. This site and domain specific knowledge lays out best practices for systems adoption such that past mistakes do not repeat themselves and new problems can be mitigated. Currently, the expectation is that innovators perform this work, yet communities can be perfectly equipped to take over performing the hard work of maintaining these systems. This involves explore what retraining programs, social support programs, technical capacity-building exercises, and regulations need to be leveraged to ensure longer term technology survival and cultural continuity. Communities will never regret including this in their proposals prior to deployment. They will regret not having done the work and being left with a broken system. This process involves planners meeting communities where they are and working with them to lay out where they need to get to in order to self-sustain their proposal, whether funded through the SCC or not.

\subsection{Build site-specificity into the process of Smart City development}

The previous four points speak to the ongoing need for site specific attention. Communities are at once resilient and fragile, unique and the same, big and small, our past and our future. Bringing together the worlds of technology, quantitative data, qualitative research, and ethnographic stories allows us to put these feelings, words, and data into plans that work for a specific community. The cohesive narrative of communities is lessened as the size of the community expands but that does not mean there is less of a story to tell, simply more stories.

SCC proposals are not simply technical exercises within the realm of engineers but have clear social and culture implications, as identified. The Biigtiigong Nishnaabeg - Pic River First Nation proposal stands out as making one of the strongest cases for the deployment of new educational service delivery methods. The strength of the case being made is largely due to the social cohesion and unified cultural narrative of the community. Communicating such a compelling narrative becomes increasingly more difficult as the scale of the community increases and higher complexity technical solutions are proposed to address broader socio-environmental issues. Multiple competing interests, stakeholder opinions, and cultural groups are all brought to bear on smart infrastructure and service delivery projects in larger cities. 
What is witnessed is that as proposals increase in magnitude they decrease in specificity. Rather than weaving together compelling narratives from a complex tapestry of characters, the socio-cultural aspect of the proposal moves towards the generic. This is exactly the planner-shaped hole that urban professionals should be filling in these projects. There is little reason for a municipality as uniquely equipped as Richmond to not be able to tell its own cultural "Great Flood Story" story (Biigtigong Nishnaabeg, 2018, p. 10). The frantic moving of people in efficient transit streams through Vancouver and Surrey or the sustainable farm to table pipeline of OCN-Kelsey are all stories untold in the SCC proposals but inherently familiar to planners. Planners, as a profession, are trained to grab knotty bundles of data, governance models, policy, social issues, and personal anecdotes in order to tease out stories that, braided together, form a unifying narrative to justify, direct, compel, and incent change through our work.

Kaner's (2019) commentary on designing the inclusive Smart City speaks to the need to design systems specifically, and not for a generic 'everyone', citing that when designing for more generalized locales it is, "far too easy to make assumptions about how 'everyone' fits into our ideal 'smart' environment" noting that the model citizen will lean towards being, "able-bodied, digitally literate, and financially stable". Kaner's argument continues by stating that blanket improvements can only go so far and, "do little to support behaviour change, citizen safety, or engagement with the city", such improvements, like upgraded Smart transit and traffic control mechanisms "end up supporting city users who are already independent of changes to city services" (Kaner, 2019).

This is an important argument, especially when examining how a narrative changes as proposals scale from communities of 400 to 1.1 million. Kaner's recommendation is to target areas of a community where services are failing citizens through extending a dialogue to citizens citing that inclusion is not just listening to everyone but, "actively taking steps to challenge and change our approaches to Smart City design". Recognition of this argument marks a transition point in Smart City development, one which the $S C C$ actively advances with proposal questions geared towards demonstrating community consultation. Boyd's (2015) modelling of Smart City generations speaks to how this model has developed over time. The Smart City 1.0 was a technology driven process focused on turning cities into highly efficient machines. This generation was rife with unsustainable private-sector driven pilot projects. The Smart City 2.0 trend was a technology-enabled, government led model which focused on broad, generalized quality of life improvements, of the kind critiqued by Kaner (2019). Interventions in this generation are the result of government procuring and applying Smart City products. Many large cities today fall within this classification but a criticism of this class of Smart City developments is the lack of community involvement. The Smart City 3.0 model is what Kaner is pointing towards. It is a trend that, like third wave sustainability itself, brings together overarching advancements in technology and the specificity of place through community co-creation models.

This can be a daunting task for communities new to this form of development, especially when dealing with the uncertainty and complexity of envisioning and developing within a Smart City context. Many municipalities of all sizes may lack the capacity to run a co-creation process themselves or the resources to fully outsource the process to a private engagement or planning firm. Initiatives like the Future Cities Canada (2019) Community Solutions Network, being led by Evergreen and OpenNorth, can begin to build Smart City development resources similar that extend co-creation thinking from the Smart Cities 
Challenge into programming that empowers communities of all shapes and sizes to own their Smart City narrative while building planning and community capacity in this realm of practice.

\section{Peace of Mind and The Long Road Ahead}

Despite moments of clarity there is no -ism to tidely conclude this research. At present, what is being advanced is an ethic of care prevalent amongst feminist literature, the extension of self-determinism to other communities that is found in indigenous technology adoption, the (in)visibility of repair \& the inequity of who determines waste found in Maintainer literature, the demanding of better processes found in social planning practice. All these themes collectively converge when critiquing Smart City-building. The unexpectedness of each of these schools of thought encountering each other on this one singular topic is compelling, with each contributing something of value that needs to be considered when addressing new infrastructure, disruptive or otherwise. The messiness is the exciting part. The work is fulfilling.

There will always be Ubers, Airbnbs, Fibes, and Sidewalks. At the same time, there will always be members of the public service, unexpected community leaders, and planners concerned with ensuring that our communities develop and change in a way that benefits the public good. Involving communities in this narrative requires holding space for them to join government and innovation actors already participating in these conversations. Sustaining innovations in cities can only be strengthened by ensuring that the future vision communities are buying into is one of their own making.

\section{Postscript}

At the time of writing, full submissions had not yet been submitted to Infrastructure Canada. This research took place from September 2018 to March 2019, prior to the full Top 20 submissions of the SCC RFP being submitted to Infrastructure Canada and prior to the announcement of the winners. As such, analysis preempts selection, investment, and deployment. It does however highlight sustainability and continuity considerations within the proposals that can strengthen proposed systems prior to build-out and increase the likelihood of longevity. No one wants to see these systems fail, least of all the public sector actors funding them and the people using them. 


\section{Appendices}

\section{Appendix 1 - Top Twenty Smart Cities Challenge Finalists}

\section{$\$ 5$ Million Prize Category}

1. Biigtigong Nishnaabeg (Pic River First Nation), Ontario

2. Cree Nation of Eastmain, Quebec

3. Town of Bridgewater, Nova Scotia

4. Mohawk Council of Akwesasne, Quebec

5. City of Yellowknife, Northwest Territories

\$50 Million Prize Category

1. Region of Waterloo, Ontario

2. Quebec City, Quebec

3. City of Edmonton, Alberta

4. City of Surrey \& City of Vancouver, BC

5. Montréal, Quebec
\$10 Million Prize Category

1. Town of The Pas, Opaskwayak Cree Nation, Rural Municipality of Kelsey, Manitoba

2. City of Côte Saint-Luc, Quebec

3. Nunavut Communities, Nunavut

4. Saint Mary's First Nation and City of Fredericton, New Brunswick

5. Parkland, Brazeau, Lac Ste Anne and Yellowhead Counties, Alberta

6. City of Airdrie and Area, Alberta

7. City of Richmond, British Columbia

8. City of Guelph \& Wellington County, Ontario

9. City of Saskatoon, Saskatchewan

10. Greater Victoria, British Columbia 


\section{Appendix 2 - Places to Intervene in a System}

Meadows (1970)

In increasing order of leverage/effectiveness:

12. Constants, parameters, numbers (such as subsidies, taxes, standards).

11. The sizes of buffers and other stabilizing stocks, relative to their flows.

10. The structure of material stocks and flows (transport networks, population age structures).

9. The lengths of delays, relative to the rate of system change.

8. The strength of negative feedback loops, relative to the impacts being corrected against.

7. The gain around driving positive feedback loops.

6. The structure of information flows (who does and does not have access to information).

5. The rules of the system (such as incentives, punishments, constraints).

4. The power to add, change, evolve, or self-organize system structure.

3. The goals of the system.

2. The mindset or paradigm out of which the system - its goals, structure, rules, delays, parameters - arises.

1. The power to transcend paradigms.

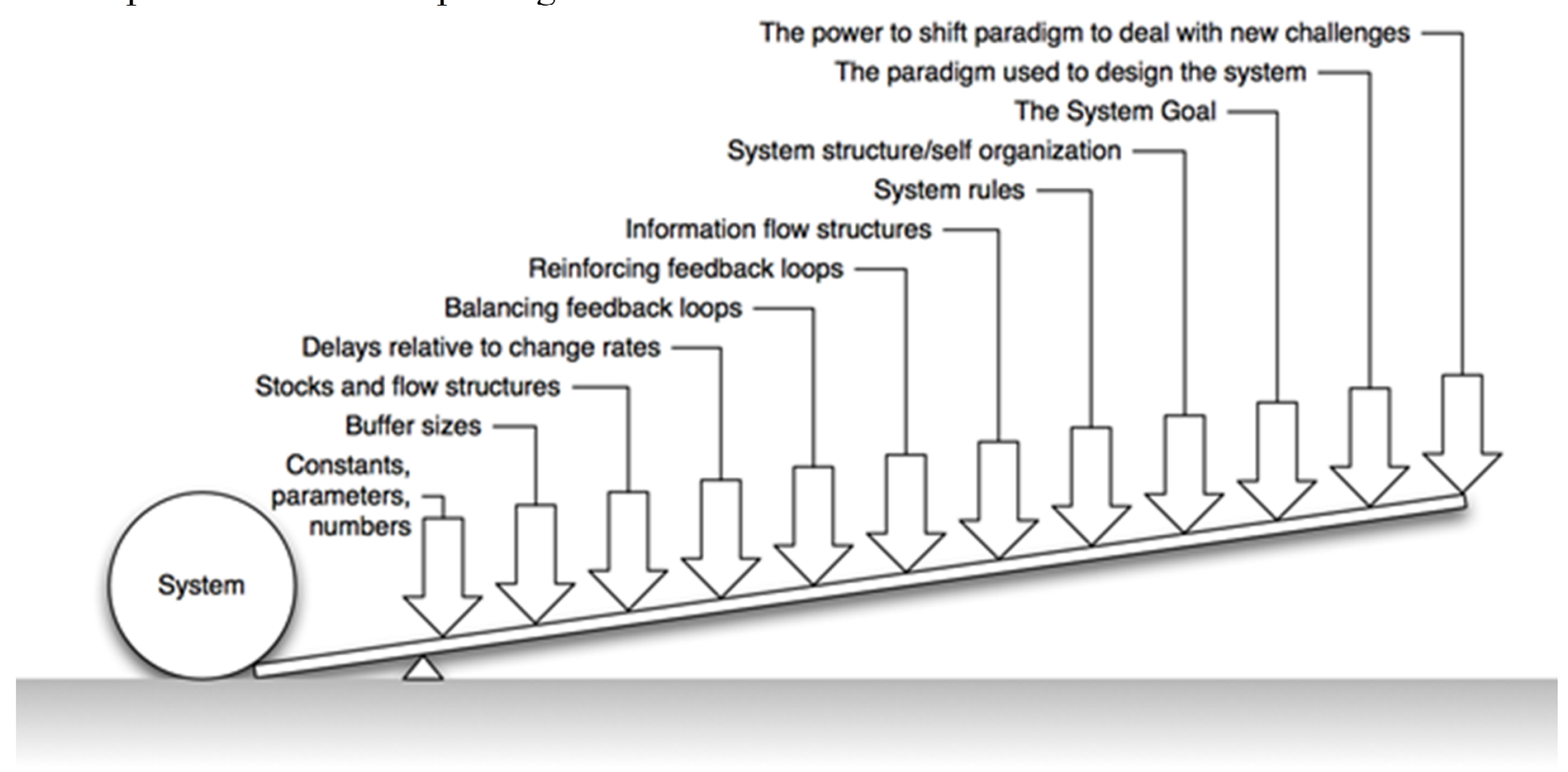




\section{Appendix 3 - Types of Systems \& Scales of Systems}

This act of understanding is further outlined by Cruz and Sarmento's (2017) assessment model for risk levels and their associative risk-adjusted return on investment:.

Business As Usual: ("BAU") is a model closely representative of traditional P3 models within which both private and public sector actors are familiar. This mode will generally maintain a "Build, Operate, Transfer" governance structure. A BAU class Smart City project does not preclude innovation from occurring within the project. Rather, use of new technological solutions will be heavily prescriptive. This class is identical to the project manager picking a "better" or more recent product over a lower performing or older product.

Incremental Innovations: Generally involve integrating a soft capital asset into an existing hard capital asset that will extend its longevity, modify the product's lifecycle, or increase the efficiency of a system. Upgrades create new learnings and efficiencies above and beyond a regular BAU upgrade. It may uncover new problems or illustrate solutions to previously unsolvable problems. This class of innovation is considered riskier than the BAU class as the product's benefits may not be fully proven at the time of integration, deployment may require significant adaptation to function in a specific site or system, and the new technology may introduce new problems to the system.

Groundbreaking Innovations: These projects represent exploratory and unproven actions that carry the possibility of becoming products or business models in the future. Groundbreaking innovations tend not to be bankable, in that they could not be funded privately at a similar level of risk. Within a groundbreaking innovation model there is no guarantee that the project will achieve substantial completion, let alone be considered a successful and useable system. 


\section{Appendix 4 - Rust, Dust, Cracks, \& Corruption}

Mattern's model (outlines product, system, and infrastructure sub-systems of wear and tear, detailing how innovators and maintainers act to ward off the natural and expected impacts of use, entropy, and internal chronic stresses. Where analysis begins to shift to higher levels and broader understandings of volatility is in how systems react to, and bounce back from acute shocks or longer term external chronic stresses.

Rust speaks to the attention required of large physical infrastructure systems as well as the social and political relationships in which such systems are entrenched. Mattern states, "maintenance traverses scales", the attention required being not just the availability of labour but also the political and economic decisions required to direct such upkeep.

Dust illustrates the interiors of these systems, exploring how we interact with the and the recursive relationship between the ability of maintainers to maintain vis-a-vis designs which teach, support, or prohibit the performance of such labour. This lens speaks as much to the hierarchical power relations that make design decisions as it does to the designs of architects and engineers.

Cracks is the fixing of objects. This lens is used to analyze discrete objects within systems and how legible they are to the performance of maintainer labour practices such as repair and modification. Whether objects can be fixed or maintained by their owners, if specialty labour is required, or if the seamless design of the object prohibits human intrusion altogether must all be considered when evaluating the low-level objects that collectively comprise higher level experiences or infrastructure.

Corruption is Mattern's final lens, which identifies the critical role of code in maintaining everything from thermostats to subway systems. Citing Ribes and Finhold, whose work argues that these systems need to be designed for the "long now" citing that software maintenance for opensource projects lacks the required financial support for dedicated staff and is reliant upon volunteer labour. Similarly, online communities and data archives both require moderators to clean up content and entries. Collectively, software is fragile with a multitude of dependencies, all of which must be maintained to the same extent as the visible, physical assets noted in prior wear and tear lenses. 


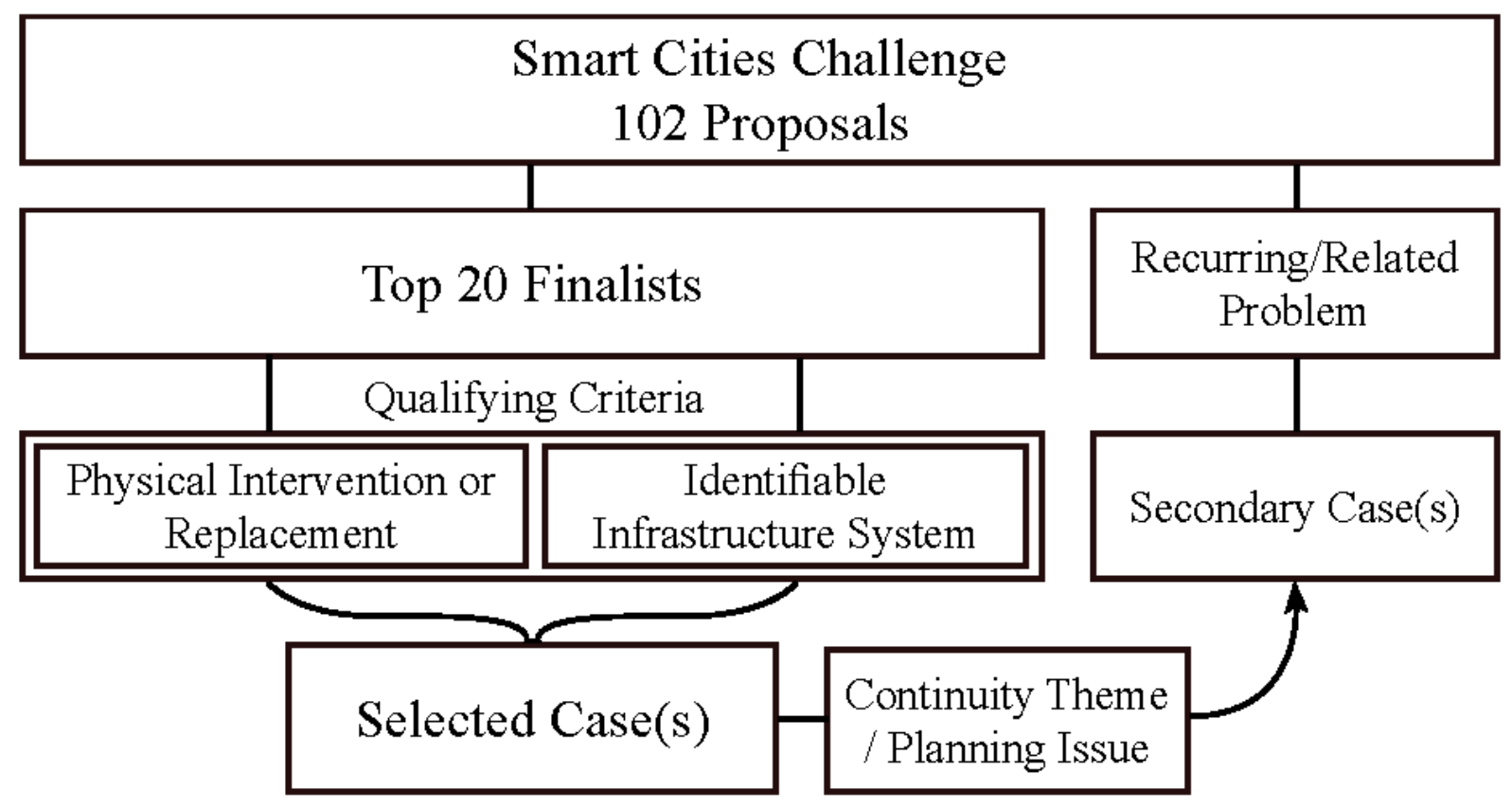




\section{Appendix 6 - SCC Entries}

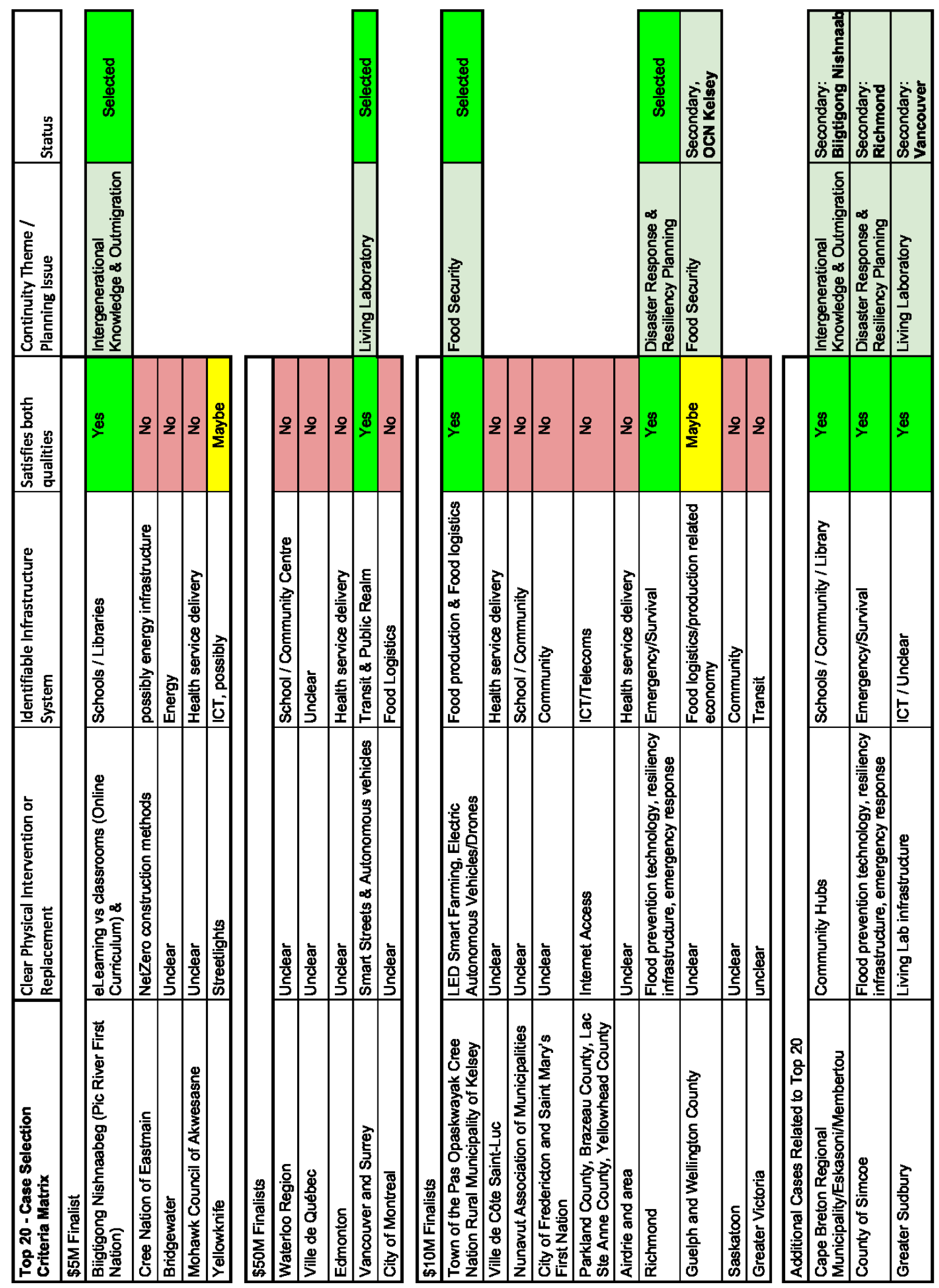




\section{Appendix 7 - City of Richmond - Proposed Emergency Response System}

This system map was developed by piecing together separate parts of the City of Richmond's Smart Cities Challenge RFP proposal. This documentation was not included in their submission package, but was developed by the other of this paper.

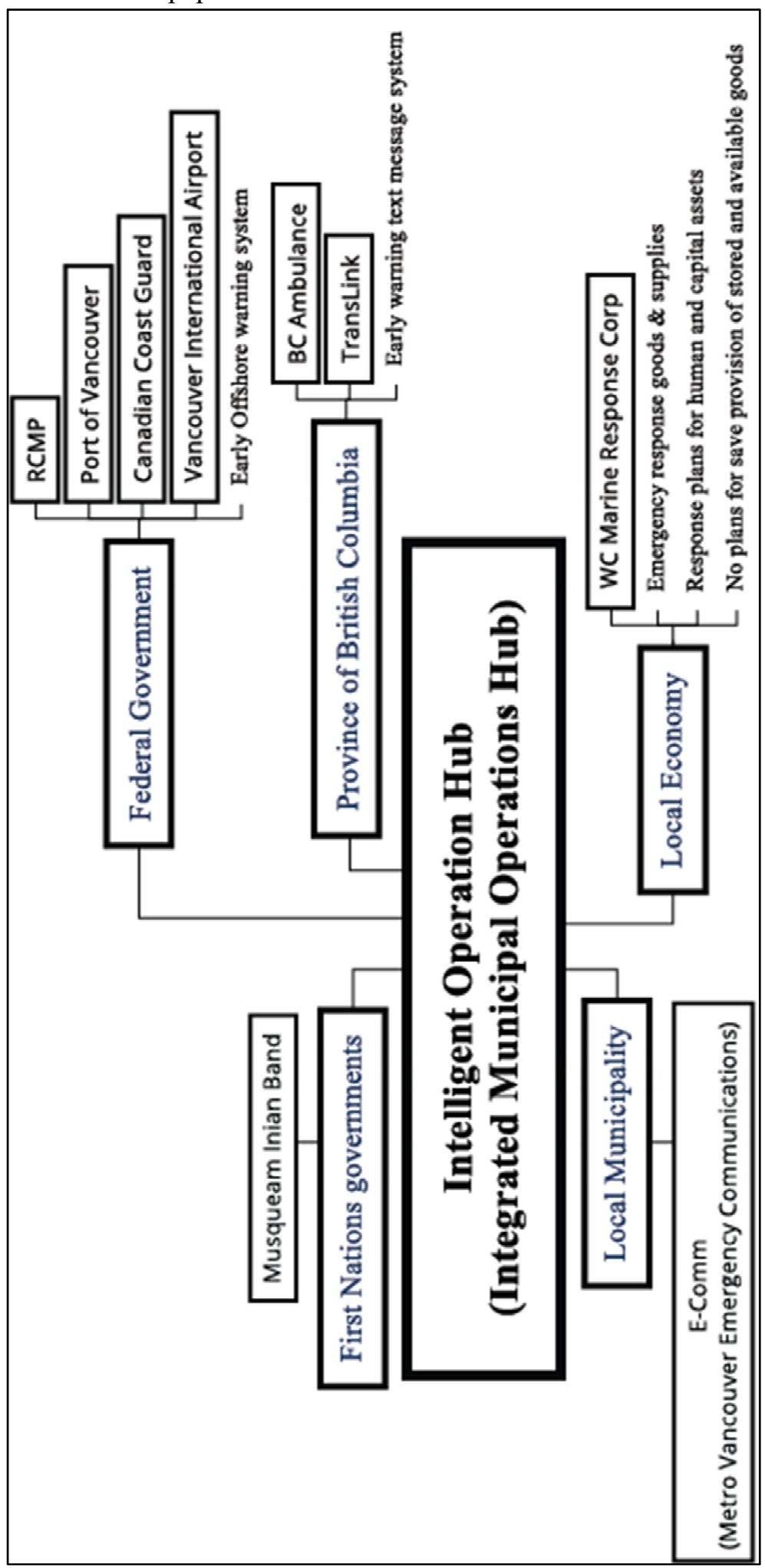




\section{Appendix 8 - Kurzweil's Model}

Kurzweil; three-stage transition model for accepting new technologies

1. "Awe and wonderment at their potential to overcome age-old problems;

2. A sense of dread at the new set of dangers

3. Realizing "the only viable and responsible path is to set a careful course to reap the benefits while managing the dangers 


\section{References}

Agro, C., Apollonio, M., \& Cheung, M. (2013). Toronto ice storm leaves 230,000 without power | CBC News. Retrieved from https://www.cbc.ca/news/canada/toronto/toronto-ice-storm-leaves-230-000without-power-1.2473543

Akrich, M. (1992). The De-scription of Technical Objects. In W. E. Bijker \& J. Law (Eds.), Shaping Technology/Building Society. Studies in Sociotechnical Change (pp. 205-224). Cambridge: MIT Press

Alizadeh, T. (2017). Urban Digital Strategies: Planning in the Face of Information Technology?. Journal Of Urban Technology, 24(2), 35-49. http://dx.doi.org/10.1080/10630732.2017.1285125

April, A., \& Abran, A. (2008). 1.2.2 Maintainers' Perceptions of Software Maintenance Issues In Software Maintenance Management. Wiley. Retrieved from https://books-scholarsportalinfo.ezproxy.lib.ryerson.ca/uri/ebooks/ebooks2/ieee/2013-04-12/1/6129685

Atkin, R. (2018). Stop replacing London's phone boxes with corporate surveillance. Retrieved from https://www.wired.co.uk/article/linkuk-bt-google-free-wifi-and-calls-london

Balsillie, J. (2018). Sidewalk Toronto has only one beneficiary, and it is not Toronto. Retrieved from https://www.theglobeandmail.com/opinion/article-sidewalk-toronto-is-not-a-smart-city/

Bannerman, S. (2019). Canadians are rightly worried about invasion of privacy in smart cities. Retrieved from https://theconversation.com/canadians-are-rightly-worried-about-invasion-of-privacy-in-smartcities-110091

Biigtigong, Nishnaabeg (Pic River First Nation). (2018). Smart Cities Challenge [Ebook]. Retrieved from http://biigtigong.com/language/publications/smart-cities-challenge/

Bijker, W. (1992). Postscript: Technology, Stability, and Social Theory In Bijker, W. et, al. Shaping technology/building society: studies in sociotechnical change.Cambridge, Mass.: MIT Press.

Bliss, L. (2016, April 11). How 'Maintainers,' Not 'Innovators,' Make the World Turn. Retrieved February 28, 2018, from https:/www.citylab.com/design/2016/04/how-maintainers-not-innovators-make-theworld-turn/477468/?utm_source=SFFB

Bliss, L. (2018). Behind the Uber Self-Driving Car Crash: a Failure to Communicate. Retrieved from https:/www.citylab.com/transportation/2018/05/behind-the-uber-self-driving-car-crash-a-failure-tocommunicate/561230/

Branchi, P., Fernández-Valdivielso, C., \& Matías, I. (2015). Urban technology analysis matrix. Management Of Environmental Quality: An International Journal, 26(3), 342-356. http://dx.doi.org/10.1108/meq-02-2014-0026

Brasuell, J. (2017). The 1854 Map That Transformed Public Health in Urban Areas. Retrieved from https://www.planetizen.com/news/2017/11/95755-1854-map-transformed-public-health-urban-areas 
Canada, Government of. (2005). ARCHIVED - Robertson Screw and Screwdriver - Incredible Inventions - Cool Canada - Library and Archives Canada. Retrieved from https://www.collectionscanada.gc.ca/cool/002027-2008-e.html

Cape Breton Regional Municipality. (2018). CBRM Smart Cities Challenge Proposal[Ebook]. Retrieved from https://docs.wixstatic.com/ugd/71542c 0e989683b2c9421897afffe086aa5ec2.pdf

Childs, K., \& Zegart, A. (2019). The Divide Between Silicon Valley and Washington Is a NationalSecurity Threat. Retrieved from https://www.theatlantic.com/ideas/archive/2018/12/growing-gulfbetween-silicon-valley-and-washington/577963/

Christensen, C. (2016). The innovator's dilemma. Boston: Harvard Business Review Press.

Christians, C. (2007). Cultural continuity as an ethical imperative. Qualitative Inquiry, 13(3), 437-444. doi:10.1177/1077800406297664

Clarke, A. (2018). The Evolving Role of Non-State Actors in Digital Era Government. Retrieved from SSRN: https://papers.ssrn.com/sol3/papers.cfm?abstract id=3268084

Cochrane, D. (2019). Federal budget to target Canada-wide high speed internet by 2030 | CBC News. Retrieved from https://www.cbc.ca/news/politics/budget-2019-internet-speed-1.5060457

Cohen, B. (2015). The 3 Generations Of Smart Cities. Retrieved from https:/www.fastcompany.com/3047795/the-3-generations-of-smart-cities

Cruz, C., \& Sarmento, J. (2017). Reforming traditional PPP models to cope with the challenges of smart cities. Competition And Regulation In Network Industries, 18(1-2), 94-114. doi: 10.1177/1783591717734794

Davis Jr, S. (2018). The How and Where of Regulating Communication Technology? In Clark, J, et al. Sovereignty: The Race to Regulate, Putting Consumers First as Communications Technology Emerges. Federal Communications Law Journal, May 2018, p. 303+. Academic OneFile, http://link.galegroup.com/apps/doc/A553314014/AONE?u=rpu_main\&sid=AONE\&xid=a8a15a48. Accessed 13 Dec. 2018.

Deacon, A. (2017). The messiness of innovation: Cities of the future will be shaped by how they balance ordered efficiency with creative disruption. UMKC Law Review 85(4), 833-844.

DeBose, C. (1992). Codeswitching: Black English and Standard English in the African-American linguistic repertoire. In Eastman, Carol. Codeswitching. Clevedon: Multilingual Matters. pp. 157167. ISBN 1-85359-167-X.

Denis, J., \& Pontille, D. (2017). Object ontologies and the contrasted in/visibilities of maintenance work . Hoboken, NJ: Maintainers II Conference. Retrieved February 28, 2018, from https://static1.squarespace.com/static/56a8e2fca12f446482d67a7a/t/58dffa90f7e0ab3ba86db004/1491 073681590/DenisPontille+Object+Ontologies+\%26+Maintenance+Work.pdf 
Dente, B., \& Coletti, P. (2011). Measuring Governance in Urban Innovation. Local Government Studies, 37(1), 43-56. http://dx.doi.org/10.1080/03003930.2010.548553

Desouza, K., \& Flanery, T. (2013). Designing, planning, and managing resilient cities: A conceptual framework. Cities, 35, 89-99. http://dx.doi.org/10.1016/j.cities.2013.06.003

Dewey, C. (2019). How Google Wiped a Neighborhood off the Map. Retrieved from https://onezero.medium.com/how-googles-bad-data-wiped-a-neighborhood-off-the-map$\underline{80 \mathrm{c} 4 \mathrm{c} 13 \mathrm{flc} 2 \mathrm{~b}}$

Dovey Fishman, T., \& Flynn, M. (2018). Using public-private partnerships to advance smart cities Part two: Funding and financing smart cities series [Ebook]. Deloitte Center for Government Insights. Retrieved from https://www2.deloitte.com/content/dam/Deloitte/global/Documents/Public-Sector/gxps-public-private-partnerships-smart-cities-funding-finance.pdf

Dushenko, W. (2012) Urban-Rural Tensions, Place, and Community Sustainability in William T. Dushenko et al. Urban Sustainability: Reconnecting Space and Place, University of Toronto Press, 2012, pp. 211-238.

Ensmenger, N. (2014) When Good Software Goes Bad - The Surprising Durability of an Ephemeral Technology. Indiana University. Retrieved from http://homes.sice.indiana.edu/nensmeng/files/ensmenger-mice.pdf

Ferreira, M., Mota de Sá, F., \& Oliveira, C. (2014). Disruption index, DI: an approach for assessing seismic risk in urban systems (theoretical aspects). Bulletin Of Earthquake Engineering, 12(4), 1431 1458. http://dx.doi.org/10.1007/s10518-013-9578-5

Foster, E. K. (2017). Systems of Maintenance: Feminist Theory and Method. Hoboken, NJ: Maintainers II Conference. Retrieved February 28, 2018, from https://static1.squarespace.com/static/56a8e2fca12f446482d67a7a/t/5705b6fa3c44dd81ea74296a2/145 9992314105/Maintainers+Text_Ellen+Foster.pdf

Fox, C. (2019). King Street restaurants say business is still suffering during transit pilot project. Retrieved from https://toronto.ctvnews.ca/king-street-restaurants-say-business-is-still-suffering-during-transitpilot-project-1.4287225

Future Cities Canada. (2019). Making the case for Open Smart Cities | Future Cities Canada. Retrieved from https://futurecitiescanada.ca/stories/making-the-case-for-open-smart$\underline{\text { cities/?utm_source }=\text { fcc\&utm medium=email\&utm_campaign=fccnews }}$

Gallagher, W. (2017). How the Post Office Created America. Penguin Books.

Gendron, A. \& Rudner, M. (2012, March) Assessing Cyber Threats to Canadian Infrastructure. Canadian Security Intelligence Service. Retrieved from: https://www.canada.ca/content/dam/csisscrs/documents/publications/CyberTrheats_AO_Booklet_ENG.pdf 
Gilbert, A. (2019). The time that Tony Fadell sold me a container of hummus. Retrieved from https://arlogilbert.com/the-time-that-tony-fadell-sold-me-a-container-of-hummuscb0941c762c1\#.tsud8t7hs

Goodspeed, R. (2016). Digital knowledge technologies in planning practice: From black boxes to media for collaborative inquiry. Planning Theory \& Practice, 17(4), 577-600.

doi:10.1080/14649357.2016.1212996

Google. (2010). Think big with a gig: Our experimental fiber network. Retrieved from https://googleblog.blogspot.com/2010/02/think-big-with-gig-our-experimental.html

Goren, M. (2016). Three Types of Construction Specification. Retrieved from https://www.pointtopoint.com/blog/three-types-of-construction-specification

Greater Sudbury, City of. (2018). Smart Cities Application [Ebook]. Retrieved from https://www.greatersudbury.ca/sudburyen/assets/File/Comms/City $\% 20$ of $\% 20$ Greater $\% 20$ Sudbury $\% 2$ 0Smart $\% 20$ Cities $\% 20$ Application $\% 20$ Online to $\% 20$ post $\% 20$ online.pdf

Griggs, T., \& Wakabayashi, D. (2018). How a Self-Driving Uber Killed a Pedestrian in Arizona. Retrieved from https:/www.nytimes.com/interactive/2018/03/20/us/self-driving-uber-pedestriankilled.html

Greenberg, K. (2017). GREENBERG: How Sidewalk Labs could make a vital contribution to Toronto's evolution - Spacing Toronto. Retrieved from http://spacing.ca/toronto/2017/11/21/greenberg-howsidewalk-labs-could-make-a-vital-contribution-to-torontos-evolution/

Grewal, J. (2019). High-speed internet coming to 200 rural B.C. communities - The Columbia Valley Pioneer. Retrieved from https:/www.columbiavalleypioneer.com/news/high-speed-internet-comingto-200-rural-b-c-communities/

Guelph, City of, \& Wellington County. (2018). Smart Cities Challenge [Ebook]. Retrieved from https://guelph.ca/wp-content/uploads/GW-SmartCities-Application.pdf

Harford, T. (2017). How the lift transformed our cities. Retrieved from https://www.bbc.com/news/business-39735801

Hartshorn, R. (2018). The Trouble With Cryptocurrency's Viral Growth. Retrieved from https://www.nytimes.com/2018/09/25/opinion/cryptocurrency-bitcoin-viral-growth.html

Heinsdorf, M. (2014). Closed and open specifications - Consulting - Specifying Engineer. Retrieved from https://www.csemag.com/articles/closed-and-open-specifications/

Higgins, T. (2019). Tesla Is Cranking Out Model 3s - Now It Has to Service Them. Retrieved from https://www.wsj.com/amp/articles/tesla-is-cranking-out-model-3snow-it-has-to-service-them11549810800

Horne, R. (2018). On Disruption, Transformation \& Dividends. Ontario Planning Journal, 33(5), 1-2. Retrieved from https://ontarioplanners.ca/OPPIAssets/Journal/Issues/33-5-Journal-FINALinteractive.pdf

Innisfil, C. (2018). Innisfil | Innisfil Transit. Retrieved from https://innisfil.ca/living/transit 
Isserman, A. M. (2007). Forecasting to learn how the world can work. In L. D. Hopkins \& M. A. Zapata (Eds.), Engaging the future: Forecasts, scenarios, plans and projects (pp. 175-197). Cambridge, MA: Lincoln Institute for Land Policy

Kaner, H. (2019). Designing for 'everyone' is not the path to an inclusive smart city. Retrieved from https://www.information-age.com/inclusive-smart-city-123474406/

Kearney, T. @thomkearny. (2019) In the policy world we are great at start-ups (pilot projects) but not very successful at scaling the good ones.@Clerk_GC@JenniferRiel \#policomm [Twitter post]. Retrieved from https://twitter.com/thomkearney/status/1098324429900337152

Klosterman, R. E. (2013). Lessons learned about planning. Journal of the American Planning Association, 79(2), 161-169. doi:10.1080/01944363.2013.882647

Kosoff, M. (2018). Uber Is Running Out of Ways to Save Itself. Retrieved from https://www.vanity fair.com/news/2018/03/uber-is-running-out-of-ways-to-save-itself

Kunstler, J. H. (1993). The Geography of Nowhere, Simon and Schuster

Kurzweil, R. (2001, March). Promise and Peril--The Deeply intertwined Poles of 21st Century Technology. Communications of the ACM, 44(3), 88. Retrieved from http://ink.galegroup.com.ezproxy.lib.ryerson.ca/apps/doc/A73233464/AONE?u=rpu_main\&sid=AO NE\&xid $=337 \mathrm{cb} 035$

Lang, D., et, al. (n.d.) Leverage Points for Sustainability Transformation, Project Overview. Leuphana University Lueneburg. Retrieved from https://leveragepoints.org/project-overview/

Leblanc, T. (2018). Sovereignty Disrupted In Clark, J, et al. Sovereignty: The Race to Regulate, Putting Consumers First as Communications Technology Emerges. Federal Communications Law Journal, May 2018, p. 303+. Academic OneFile, Retrieved from http://ink.galegroup.com/apps/doc/A553314014/AONE?u=rpu_main\&sid=AONE\&xid=a8a15a48..

Lepore, J. (2018). What the Gospel of Innovation Gets Wrong. Retrieved from https:/www.newyorker.com/magazine/2014/06/23/the-disruption-machine

Lepore, J. (2019). A New Americanism. Retrieved from https://www.foreignaffairs.com/articles/unitedstates/2019-02-05/new-americanism-nationalism-jill-lepore

Lewis, M. (2018). The fifth risk. New York: W. W. Norton \& Company.

Lewis, P. (1995). The Urban Invasion of Rural America, the emergence of the galactic city. The Changing American Countryside: Rural People and Places. Series: Rural America. E. N. Castle. Lawrence, University Press of Kansas: 39-62. 
Lister, N. (2012). Map-Making as Place-Making: Building Social Capital for Urban Sustainability In Dushenko, W, et al. Urban Sustainability: Reconnecting Space and Place, University of Toronto Press, 2012.

Mattern, S. (2014). Libraries As Infrastructure: Reading room, social service center, innovation lab. How far can we stretch the public library?. Retrieved from https://placesjournal.org/article/library-asinfrastructure/

Mattern, S. (2018). Maintenance and Care: A working guide to the repair of rust, dust, cracks, and corrupted code in our cities, our homes, and our social relations. Retrieved from https://placesjournal.org/article/maintenance-and-care/?cn-reloaded=1

Meadows, D. (1970). Leverage Points: Places to Intervene in a System. Retrieved February 04, 2018, from http://donellameadows.org/archives/leverage-points-places-to-intervene-in-a-system/

Meadows, D. (2008). Thinking in systems (p. 5). White River Junction, Vermont: Chelsea Green Publishing.

Meyerson, H. (2018). Operation decentralize: how small towns can save america. Walnut Creek: John Marr Press.

Nelson, G. (2018). Federal, State, and Self-Regulation Strategies for Data Collection \& Use In Clark, J, et al. Sovereignty: The Race to Regulate, Putting Consumers First as Communications Technology Emerges. Federal Communications Law Journal, May 2018, p. 303+. Academic OneFile, http://link.galegroup.com/apps/doc/A553314014/AONE?u=rpu_main\&sid=AONE\&xid=a8a15a48. Accessed 20 Dec. 2018.

Noble, M. (2018). New Technologies and Toronto's Transit Pilot . Ontario Planning Journal, 33(5), 4. Retrieved from https://ontarioplanners.ca/OPPIAssets/Journal/Issues/33-5-Journal-FINALinteractive.pdf

OECD (2018), The Innovation System of the Public Service of Canada, OECD Publishing, Paris, https://doi.org/10.1787/9789264307735-en. Retrieved from http://www.oecd.org/gov/innovative-government/innovation-system-canada-2018-highlights-en.pdf

O'Neil, C. (2016). Weapons of math destruction. London: Penguin Books.

Ontario, Government of. (2019). Planning Act, as amended. R.S.O. 1990, c.

Ontario, Government of. Ministry of Municipal Affairs and Housing. (2014). Provincial Policy Statement 2014. Toronto: Ministry of Municipal Affairs and Housing.

Ontario Professional Planners Institute (OPPI). (2012). By-law No. 1 The General By-law of the Ontario Professional Planners Institute [Ebook] (pp. 13-16). Toronto: OPPI. Retrieved from https://ontarioplanners.ca/OPPIAssets/Documents/OPPI/OPPI-BYLAW.pdf 
Pagliaro, J. (2019). Library seeks funding to expand popular youth hubs | The Star. Retrieved from https:/www.thestar.com/news/city hall/2018/02/02/library-seeks-funding-to-expand-popular-youthhubs.html

Pandey, E. (2019). The trouble with smart cities. Retrieved from https://www.axios.com/smart-citiestoronto-sidewalk-labs-las-vegas-cb0451e5-0b98-46c4-9ba3-4a7a04eb4ca7.html

Pas, Town of the, \& Opaskwayak Cree Nation, \& Kelsey, Rural Municipality of. (2018). OCN Smart Cities Challenge (Thread Title) [Ebook]. Retrieved from http://ocnsmart.freeforums.net/thread/4/

Patton, J. (2018). What is 'ShotSpotter'? Controversial gunshot detector technology approved by Toronto police. Retrieved from https://globalnews.ca/news/4344093/controversial-gunshot-detectorshotspotter-toronto-police/

Peat, D. (July 2, 2015a). Toronto taxis doomed if uber allowed to continue: Fleet operator. The Star. Retrieved from http://ezproxy.lib.ryerson.ca/login?url=https://search-proquestcom.ezproxy.lib.ryerson.ca/docview/2171005967?accountid=13631

Pic River Development Corporation. (2019). Internet Packages. Retrieved from http://prdc.picriver.com/intpackages

Pic River FN. (2018). Ojibways of the Pic River First Nation. Retrieved from http://www.picriver.com/index.pl?page=97\&top $=27$

Price Waterhouse Coopers (PwC), \& Urban Land Institute (ULI). (2018). Emerging Trends in Real Estate 2019 (p. 3). Washington, D.C.: Urban Land Institute.

Renzetti, E. (2019). Toronto is building a Sidewalk, and no one knows where it goes. Retrieved from https://www.theglobeandmail.com/opinion/article-toronto-is-building-a-sidewalk-and-no-one-knowswhere-itgoes/?utm_medium=Referrer: + Social + Network $+/+$ Media\&utm campaign=Shared + Web + Article + Li $\underline{\mathrm{nks}}$

Ribes, D. Finholt, T. A. (2009) "The Long Now of Technology Infrastructure: Articulating Tensions in Development," Journal of the Association for Information Systems: Vol. 10 : Iss. 5 , Article 5. Available at: https://aisel.aisnet.org/jais/vol10/iss5/5

Richmond, City of. (2018). Smart Cities Challenge Submission [Ebook]. Richmond. Retrieved from http://smartcity.richmond.ca/wp-content/uploads/pdf/Smart-Cities-Challenge-SubmissionMay\%202018.pdf

Robidoux, M. A., \& Mason, C. W. (2017). A Land Not Forgotten : Indigenous Food Security and LandBased Practices in Northern Ontario. Winnipeg, Manitoba: University of Manitoba Press. Retrieved from http://ezproxy.lib.ryerson.ca/login?url=https://search.ebscohost.com/login.aspx?direct=true \&db=nleb $\underline{\mathrm{k} \& \mathrm{AN}=1496877 \& \text { site }=\text { ehost-live }}$

Rosner, D. K., \& Ames, M. G. (2014). Designing for Repair? Infrastructures and Materialities of Breakdown. Proceedings of the 17th ACM conference on Computer supported cooperative work \& social computing. p. 319-331. 
Russell, A., \& Vinsel, L. (2016). Hail The Maintainers - Innovation is overvalued. Maintenance often matters more. Retrieved from https://aeon.co/essays/innovation-is-overvalued-maintenance-oftenmatters-more

Russell, A. L. \& Vinsel, L. (2018). After Innovation, Turn to Maintenance. Technology and Culture 59(1), 1-25. Johns Hopkins University Press. Retrieved December 16, 2018, from Project MUSE database https://muse-jhu-edu.ezproxy.lib.ryerson.ca/article/692165/pdf

Russell, A. L. \& Vinsel, L. (2019). Opinion | The Joy of Standards. Retrieved from https://www.nytimes.com/2019/02/16/opinion/sunday/standardization.html\#click=https://t.co/bn1Ksv $\underline{\mathrm{YchS}}$

Saval, N. (2019). Uber and the Ongoing Erasure of Public Life. Retrieved from https://www.newyorker.com/culture/dept-of-design/uber-and-the-ongoing-erasure-of-publiclife/amp? twitter impression=true

Simcoe County. (2018). Smart Cities Application [Ebook]. Retrieved from https:/www.simcoe.ca/TransportationEngineering/Documents/Smart\%20Cities\%20Application\%20\%20Final.pdf

Smeekes, A., \& Verkuyten, M. (2014). When national culture is disrupted: Cultural continuity and resistance to Muslim immigrants. Group Processes \& Intergroup Relations, 17(1), 45-66. https://doi.org/10.1177/1368430213486208

Solman, P. (2018). Cheap power drew bitcoin miners to this small city. Then came the backlash. Retrieved from https://www.pbs.org/newshour/show/cheap-power-drew-bitcoin-miners-to-this-smallcity-then-came-the-backlash

Sorbara, G. (2019). Driving my Tesla in Toronto one winter night took a little strategy and a lot of shivering in the dark | The Star. Retrieved from https://www.thestar.com/opinion/2019/02/08/drivingmy-tesla-in-toronto-one-winter-night-took-a-little-strategy-and-a-lot-of-shivering-in-the-dark.html

Start.ca. (2019). High-speed cable internet and DSL throughout Ontario | start.ca. Retrieved from https://www.start.ca/services/high-speed-internet

Statt, N. (2016). Nest is permanently disabling the Revolv smart home hub. Retrieved from https://www.theverge.com/2016/4/4/11362928/google-nest-revolv-shutdown-smart-home-products

Taneja, H. (2019). The Era of "Move Fast and Break Things" Is Over. Retrieved from https://hbr.org/2019/01/the-era-of-move-fast-and-break-things-is-over

Taylor, C., Appiah, K.A., Habermas, J., Rockefeller, S.C., Walzer, M. , \& Wolf, S. (1994). Multiculturalism: Examining the politics of recognition . Princeton, NJ: Princeton University Press.

Thompson, D. (2018a). Airbnb and the Unintended Consequences of 'Disruption'. Retrieved from https:/www.citylab.com/life/2018/02/airbnb-and-the-unintended-consequences-ofdisruption/553862/ 
Thompson, D. (2018b). Amazon's HQ2 Spectacle Isn't Just Shameful—It Should Be Illegal. Retrieved from https://www.theatlantic.com/ideas/archive/2018/11/amazons-hq2-spectacle-should-be$\underline{\text { illegal/575539/ }}$

Tien, J. (2005). Viewing urban disruptions from a decision informatics perspective. Journal Of Systems Science And Systems Engineering, 14(3), 257-288. http://dx.doi.org/10.1007/s11518-006-0194-7

Toronto (City of) v. Uber Canada Inc. et. al (Ontario Superior Court of Justice July 03, 2015).

Townsend, A. (2014). Smart cities. New York: W.W. Norton \& Company.

Uber. (2019). Innisfil | Ride | Uber. Retrieved from https://www.uber.com/cities/innisfil/ride/

Vancouver, City of, \& Surrey, City of. (2018). Smart Cities Challenge Application[Ebook]. Retrieved from https://www.smartertogether.ca/wp-content/uploads/2018/12/smart-cities-challengeapplication.pdf

Viale Pereira, G., Cunha, M., Lampoltshammer, T., Parycek, P., \& Testa, M. (2017). Increasing collaboration and participation in smart city governance: a cross-case analysis of smart city initiatives. Information Technology For Development, 23(3), 526-553.

http://dx.doi.org/10.1080/02681102.2017.1353946

Viticci, F. (2018). Erasing Complexity: The Comfort of Apple's Ecosystem. Retrieved from https://www.macstories.net/stories/apple-ecosystem-comfort/

Voigt, R. (2018). Siren Songs of the Techno-future. Ontario Planning Journal, 33(5), 22. Retrieved from https://ontarioplanners.ca/OPPIAssets/Journal/Issues/33-5-Journal-FINAL-interactive.pdf

Welch, C. (2019). Google Fiber is leaving Louisville in humiliating setback. Retrieved from https://www.theverge.com/2019/2/7/18215743/google-fiber-leaving-louisville-service-ending

Whyte, W. H., Jr.. (1980). The social life of small urban spaces. Washington, D.C. Conservation Foundation,

Winter, J., \& Boudreau, J. (2018). Supporting self-determined indigenous innovations: Rethinking the digital divide in canada. Technology Innovation Management Review, 8(2), 38-48. Retrieved from http://ezproxy.lib.ryerson.ca/login?url=https://search-proquestcom.ezproxy.lib.ryerson.ca/docview/2035667161?accountid $=13631$

Wylie, B. (2018). Searching for the Smart City's Democratic Future. Retrieved from https://www.cigionline.org/articles/searching-smart-citys-democratic-future

Zalewski, M. (2019). Illinois General Assembly - Bill Status for HB2575. Retrieved from http://www.ilga.gov/legislation/BillStatus.asp?DocNum=2575\&GAID=15\&DocTypeID=HB\&Sessio $\underline{\mathrm{nID}}=108 \& \mathrm{GA}=101$ 